\title{
Stokes diagnostics of simulated solar magneto-convection
}

\author{
S. Shelyag ${ }^{1,2}$, M. Schüssler ${ }^{2}$, S. K. Solanki ${ }^{2}$, and A. Vögler ${ }^{2}$ \\ 1 Solar Physics and Space Plasma Research Centre, Department of Applied Mathematics, University of Sheffield, Hicks Building, \\ Hounsfield Rd, S3 7RH, UK \\ 2 Max-Planck-Institut für Sonnensystemforschung, 37191 Katlenburg-Lindau, Germany \\ e-mail: msch@linmpi.mpg.de
}

Received 27 November 2006 / Accepted 16 March 2007

\begin{abstract}
We present results of synthetic spectro-polarimetric diagnostics of radiative MHD simulations of solar surface convection with magnetic fields. Stokes profiles of Zeeman-sensitive lines of neutral iron in the visible and infrared spectral ranges emerging from the simulated atmosphere have been calculated in order to study their relation to the relevant physical quantities and compare with observational results. We have analyzed the dependence of the Stokes- $I$ line strength and width as well as of the Stokes- $V$ signal and asymmetries on the magnetic field strength. Furthermore, we have evaluated the correspondence between the actual velocities in the simulation with values determined from the Stokes- $I$ (Doppler shift of the centre of gravity) and Stokes- $V$ profiles (zero-crossing shift). We confirm that the line weakening in strong magnetic fields results from a higher temperature (at equal optical depth) in the magnetic flux concentrations. We also confirm that considerable Stokes- $V$ asymmetries originate in the peripheral parts of strong magnetic flux concentrations, where the line of sight cuts through the magnetopause of the expanding flux concentration into the surrounding convective donwflow.
\end{abstract}

Key words. Sun: atmosphere - Sun: faculae, plages - Sun: magnetic fields - Sun: infrared - line: profiles - polarization

\section{Introduction}

Measurements of the photospheric magnetic fields of the Sun are mostly based on polarimetric observations of spectral lines (for an overview, see Solanki 1993; Solanki et al. 2006). In particular, two line pairs of neutral iron (Fe I), one in the visible wavelength range at $630.15 \mathrm{~nm}$ (effective Landé factor $g_{\mathrm{eff}}=1.66$ ) and $630.25 \mathrm{~nm}(g=2.5)$, and one in the infrared at $1564.85 \mathrm{~nm}$ $(g=3)$ and $1565.28 \mathrm{~nm}\left(g_{\mathrm{eff}}=1.53\right)$ have often been used for polarimetric studies since they are not blended by other lines, have large Landé factors, and because the small wavelength separation of each pair permits simultaneous observations with spectrographs (e.g., Stenflo et al. 1987b; Rabin 1992; Solanki et al. 1992; Lites et al. 1996; Martínez Pillet et al. 1997; Sigwarth et al. 1999; Stolpe \& Kneer 2000; Socas-Navarro \& Lites 2004). For magnetic field diagnostics in the visible spectral range, the Fe I lines at $630.15 \mathrm{~nm}$ and $630.25 \mathrm{~nm}$ have the advantage of being less temperature sensitive than the other widely used line pair $524.7 \mathrm{~nm}$ and $525.0 \mathrm{~nm}$ (e.g., Stenflo 1973; Wiehr 1978; Stenflo et al. 1987a; Grossmann-Doerth et al. 1996). Compared to the line pair in the visible, the infrared lines considered here are formed deeper in the atmosphere. Infrared spectral lines with sufficiently large Landé factors are well suited for the diagnostics of weak magnetic fields since the Zeeman splitting grows proportionally to the square of the wavelength while the Doppler width increases only linearly.

In this paper we carry spectro-polarimetric diagnostics of 3D radiative MHD simulations obtained with the MURaM code (Vögler et al. 2003; Vögler et al. 2005), concentrating on the lines Fe I $630.25 \mathrm{~nm}$ in the visible and Fe I $1564.85 \mathrm{~nm}$ in the infrared. While the analysis of Khomenko et al. (2005a,b) considered mixed-polarity regions with zero net vertical flux through the computational box, our study is based upon two simulations with non-vanishing net flux corresponding to horizontally averaged magnetic field strengths of $200 \mathrm{G}$ and $10 \mathrm{G}$, respectively. These simulations are considered to represent small parts of active regions in early (plage) and late stages, respectively, of their development. We calculate synthetic profiles of the Stokes parameters for the visible and infrared Fe I lines based upon the physical quantities in the simulated solar photosphere, analyze their relation to the atmospheric structure, and compare them with observational data. In particular, we statistically analyze the origin of asymmetries of Stokes $V$ profiles originating from different regions in the computational box and relate them to the velocity and magnetic field profiles along the corresponding lines of sight.

The paper is organized as follows. Section 2 gives a brief description of the simulations underlying our analysis. The line synthesis on the basis of the simulation data is explained in Sect. 3. The results are presented and discussed in Sect. 4, and Sect. 5 summarizes our conclusions.

\section{MHD simulations}

The MURaM code (Vögler 2003; Vögler et al. 2005) integrates the system of MHD equations on a three-dimensional, equidistant cartesian grid. The code is parallelized using a domain decomposition scheme. The upper boundary of the computational domain is assumed to be closed. It is located in the upper photosphere near the temperature minimum, where the density is rather low, so that the influence of the closed boundary on the dynamics of the granulation is negligible. The lower boundary is located in the convectively unstable layers of the upper convection zone. An open boundary is implemented in order to allow free motions of the fluid through the boundary. The code includes non-grey (opacity binning) radiative transport and 

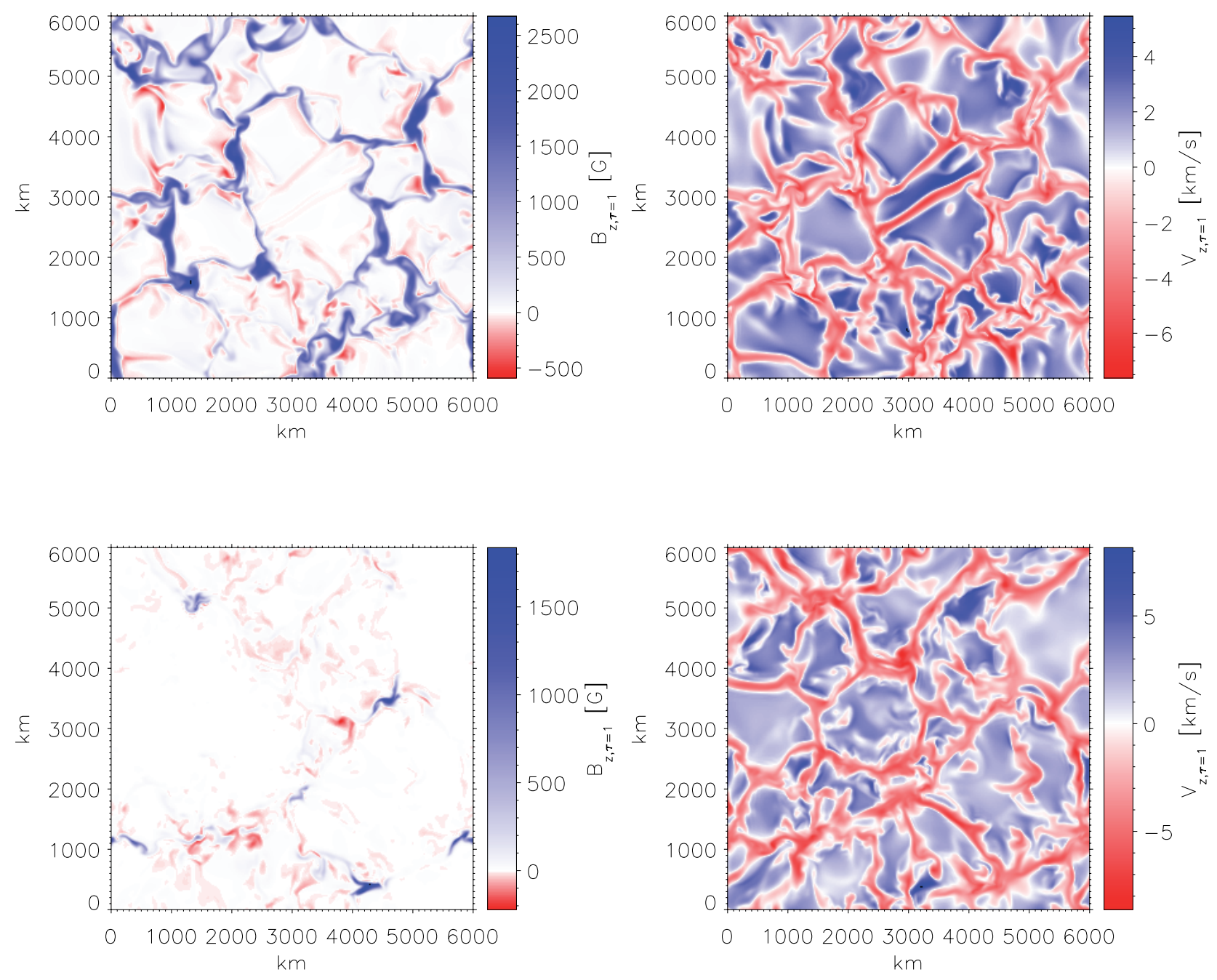

Fig. 1. Vertical magnetic field component (left panels) and vertical velocity (right panels) on the surface $\tau_{500}=1$ (continuum optical depth unity at $500 \mathrm{~nm}$ wavelength) for snapshots from simulations with $\left\langle B_{z}\right\rangle=200 \mathrm{G}$ (plage, upper row) and $\left\langle B_{z}\right\rangle=10 \mathrm{G}$ (decay phase, bottom row). Positive values of the velocity (shown in blue) correspond to upflows; downflows are are indicated in red. Note the different scales for positive and negative $B_{z}$ in the left panel (all negative-polarity field is rather weak).

an equation of state taking into account partial ionization of the 11 most abundant chemical elements in the solar photosphere.

The size of the computational domain for the simulations considered here is $6000 \times 6000 \times 1400 \mathrm{~km}^{3}$ with a grid size of $288 \times 288 \times 100$ grid cells. The corresponding resolution is $20.8 \mathrm{~km}$ in the horizontal directions and $14 \mathrm{~km}$ in the vertical. The simulation starts with a plane-parallel atmosphere extending between $800 \mathrm{~km}$ below and $600 \mathrm{~km}$ above the average level of continuum optical depth unity at $500 \mathrm{~nm}$. After convection has developed and both the outgoing energy flux and the total kinetic energy have reached stationary values (with only shortterm fluctuations on the granulation time scale), a homogeneous vertical magnetic field of $200 \mathrm{G}$ and $10 \mathrm{G}$, respectively, has been imposed. After the decay of all transients caused by the introduction of the magnetic field, we have continued the simulations for about another hour solar time to be sure that the results have become independent of the initial distribution of magnetic flux.

The snapshot from the $200 \mathrm{G}$ run (representing a small part of a plage region) is taken about 160 min after the start of simulation and 95 min after the vertical magnetic field was introduced. The snapshot from the $10 \mathrm{G}$ run (late decay phase of an active region, or base of a coronal hole) is taken about $165 \mathrm{~min}$ after the start of the simulation and $45 \mathrm{~min}$ after the vertical magnetic field was introduced. Maps of the vertical components of magnetic field and flow velocity on the surface $\tau_{500}=1$ for the two simulation snapshots are shown in Fig. 1. They illustrate the concentration of the magnetic flux in the intergranular downflow lanes and the formations of flux sheets (mainly in the $200 \mathrm{G}$ snapshot) and tube-like magnetic structures.

\section{Line synthesis}

We have calculated Stokes profiles for the FeI lines at $630.15 \mathrm{~nm}, 630.25 \mathrm{~nm}, 1564.85 \mathrm{~nm}$ and $1565.29 \mathrm{~nm}$. The main properties of the transitions corresponding to these lines, such as the central wavelength $\left(\lambda_{0}\right)$, the transition term, the effective Landé factor $\left(g_{\text {eff }}\right)$, the excitation potentials of the lower level $\left(\chi_{\mathrm{e}}\right)$, and the weighted logarithmic oscillator strengths $\left(\log g^{*} f\right)$ are given in Table 1 . We have used a value of $\epsilon=7.43$ for the iron abundance (e.g., Shchukina \& Trujillo Bueno 2001; Bellot Rubio \& Borrero 2002; Asplund et al. 2000b). The $\log \left(g^{*} f\right)$ values for the infrared lines have been taken from Borrero et al. (2003). For the $630.15 \mathrm{~nm}$ line we use the value given by Bard et al. (1991) while for $630.25 \mathrm{~nm}$ we use the same value as previous authors (e.g. Cabrera Solana et al. 2005; Khomenko et al. 2005a,b; Borrero et al. 2006). 
Table 1. Parameters of the spectral lines considered in this study.

\begin{tabular}{ccccc}
\hline \hline$\lambda_{0}[\mathrm{~nm}]$ & Transition & $g_{\text {eff }}$ & $\chi_{\mathrm{e}}[\mathrm{eV}]$ & $\log \left(g^{*} f\right)$ \\
\hline 630.15 & $\mathrm{z}^{5} \mathrm{P}_{2}^{0}-\mathrm{e}^{5} \mathrm{D}_{2}$ & 1.66 & 3.654 & -0.718 \\
630.25 & $\mathrm{z}^{5} \mathrm{P}_{1}^{0}-\mathrm{e}^{5} \mathrm{D}_{0}$ & 2.5 & 3.686 & -1.235 \\
1564.85 & $\mathrm{e}^{7} \mathrm{D}_{1}-3 \mathrm{~d}^{6} 4 \mathrm{~s} 5 \mathrm{p}^{7} \mathrm{D}_{1}^{0}$ & 3 & 5.43 & -0.675 \\
1565.29 & $\mathrm{f}^{7} \mathrm{D}_{5}-(9 / 2)[7 / 2]_{4}^{0}$ & 1.53 & 6.25 & -0.043 \\
\hline
\end{tabular}

The line profile calculations have been carried out by STOPRO routine (Solanki 1987; Frutiger 2000). It computes the full Stokes vector by integrating the radiative transfer equations under the assumption of local thermodynamic equilibrium (LTE), including Zeeman splitting and magneto-optical effects. In order to obtain quantities required for line profile calculations which are not delivered directly by the MURaM result files, the MODCON routine was used. It determines the LTE ionization equilibrium for a given chemical composition and derives electron pressure, continuum optical depth, and continuum absorption coefficient.

We have developed the program LINE, which calculates the Stokes parameters for the 3D atmosphere simulated by the MURaM code. The program uses STOPRO and MODCON as subroutines. The program was parallelized for computational efficiency of the line profile calculations in large 3D computational domains.

\section{Results of stokes diagnostics}

\subsection{General properties}

We have calculated Stokes profiles for both pairs of Fe I lines, taking vertical lines of sight through each of the $288 \times 288$ horizontal resolution elements of the computational box.

In order to demonstrate the general consistency of synthetic line profiles from simulations with observations, we show in Fig. 2 a comparison of the spatially averaged Stokes- $I$ profiles of the Fe I line pairs in the infrared (upper panel) and in the (lower panel) from the $\left\langle B_{z}\right\rangle=10 \mathrm{G}$ simulation (diamond symbols) with the observed spectrum of the quiet Sun (Delbouille et al. 1973, black curves). Observed and simulated line profiles agree to within a few percent. A more detailed comparison of mean profiles would require considering a large number of snapshots from the simulation run covering several periods of the 5-min p-mode oscillation, which is beyond the scope of this paper (see, e.g., Asplund et al. 2000a).

In what follows, we shall concentrate on the results for one line in the visible $(630.25 \mathrm{~nm}$, for simpler notation abbreviated to $630.2 \mathrm{~nm}$ in what follows) and one line in the infrared $(1564.85 \mathrm{~nm}$, abbreviated to $1564.8 \mathrm{~nm})$. We define a number of useful quantities to describe the properties of the synthetic profiles, namely, the line strength,

$S=\frac{1}{\lambda_{0}} \int \frac{\left(I_{\mathrm{c}}-I\right)}{I_{\mathrm{c}}} \mathrm{d} \lambda$,

where $I$ is the (wavelength-dependent) intensity in the line, $I_{\mathrm{c}}$ the continuum intensity, and $\lambda_{0}$ the wavelength of the line center in the rest frame. It is clear from the definition that the line strength is equal to the equivalent width normalized by the central wavelength. Furthermore, we consider the unsigned Stokes-Varea, defined as

$A_{V}=\int \frac{|V|}{I_{\mathrm{c}}} \mathrm{d} \lambda$
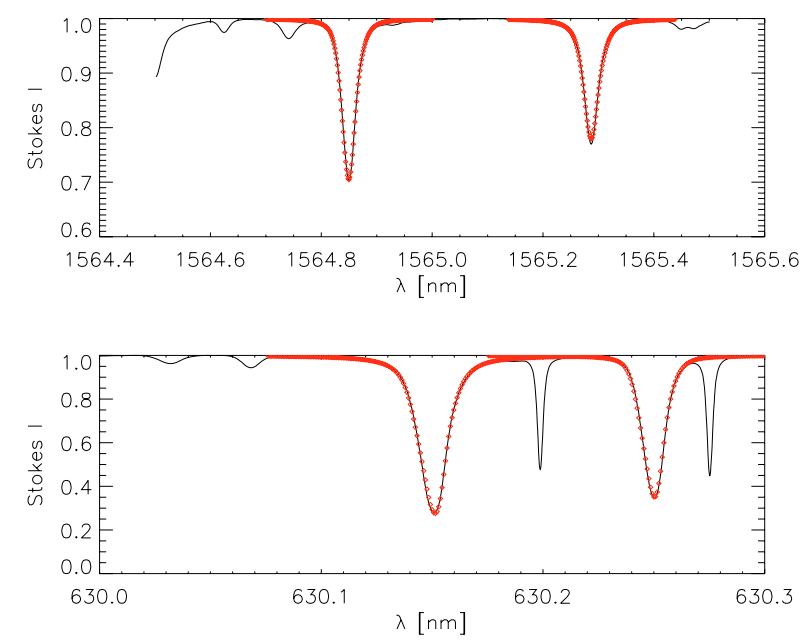

Fig. 2. Comparison between the spatially averaged synthetic Fe I line profiles for the simulation with $\left\langle B_{z}\right\rangle=10 \mathrm{G}$ (diamond symbols) and observed quiet-Sun profiles (solid curves) from the spectral atlas of Delbouille et al. (1973).

where $V$ is the Stokes $V$ parameter, and the Stokes-Varea asymmetry $^{1}$

$\delta A_{V}=\frac{\int V \mathrm{~d} \lambda}{\int|V| \mathrm{d} \lambda}$,

as well as the Stokes-V amplitude asymmetry, defined as

$\delta a_{V}=\left(\left|a_{b}\right|-\left|a_{r}\right|\right) /\left(\left|a_{b}\right|+\left|a_{r}\right|\right)$,

where $a_{b}$ is the amplitude of the "blue" lobe of the Stokes- $V$ profile and $a_{r}$ is the corresponding quantity of the "red" lobe. In all cases above, the integrals are taken over the full width of the line.

Figure 3 shows some results obtained for the snapshot from the $\left\langle B_{z}\right\rangle=200 \mathrm{G}$ (plage) run. The normalized continuum image for $\lambda=630.2 \mathrm{~nm}$ shows localized brightness enhancements corresponding to the concentrations of strong vertical magnetic field. The latter are clearly detectable in the maps of the unsigned Stokes- $V$ area (middle left panel for the $630.2 \mathrm{~nm}$ line, bottom left panel for $1564.8 \mathrm{~nm}$ ) as can be seen by comparing with the magnetic field map in the top left panel of Fig. 1. While the unsigned Stokes- $V$ area in both lines follows the total field strength rather well, the larger Zeeman sensitivity of the $1564.8 \mathrm{~nm}$ infrared line is clearly reflected in the maps, more than compensating for its smaller line strength. The larger $A_{V}$ value of this line may partly also result from its lower formation height and from the fact that strong flux concentrations show a larger height gradient of the field strength (compare the magnetic field maps in Fig. 1 with those in Figs. 7 and 8). The corresponding maps of the line strength (top middle and top right panels, respectively) reveal significant line weakening in the regions of strong magnetic field. The maps of the Stokes- $V$ area asymmetry (central panel and bottom middle panel, respectively) and of the amplitude asymmetry (middle right and bottom right panel, respectively) show strong asymmetries in the periphery of magnetic flux concentrations, while their central parts exhibit almost no

1 The sign of the net magnetic flux through the simulation box has been chosen such that, for a normal two-lobed $V$-profile, $\delta A_{V}$ is equal to the result of the usual definition $\left(\left|A_{b}\right|-\left|A_{r}\right|\right) /\left(\left|A_{b}\right|+\left|A_{r}\right|\right)$, where $A_{b}$ and $A_{r}$ are the areas of the "blue" and "red" lobes, respectively (e.g., Solanki \& Stenflo 1984). 

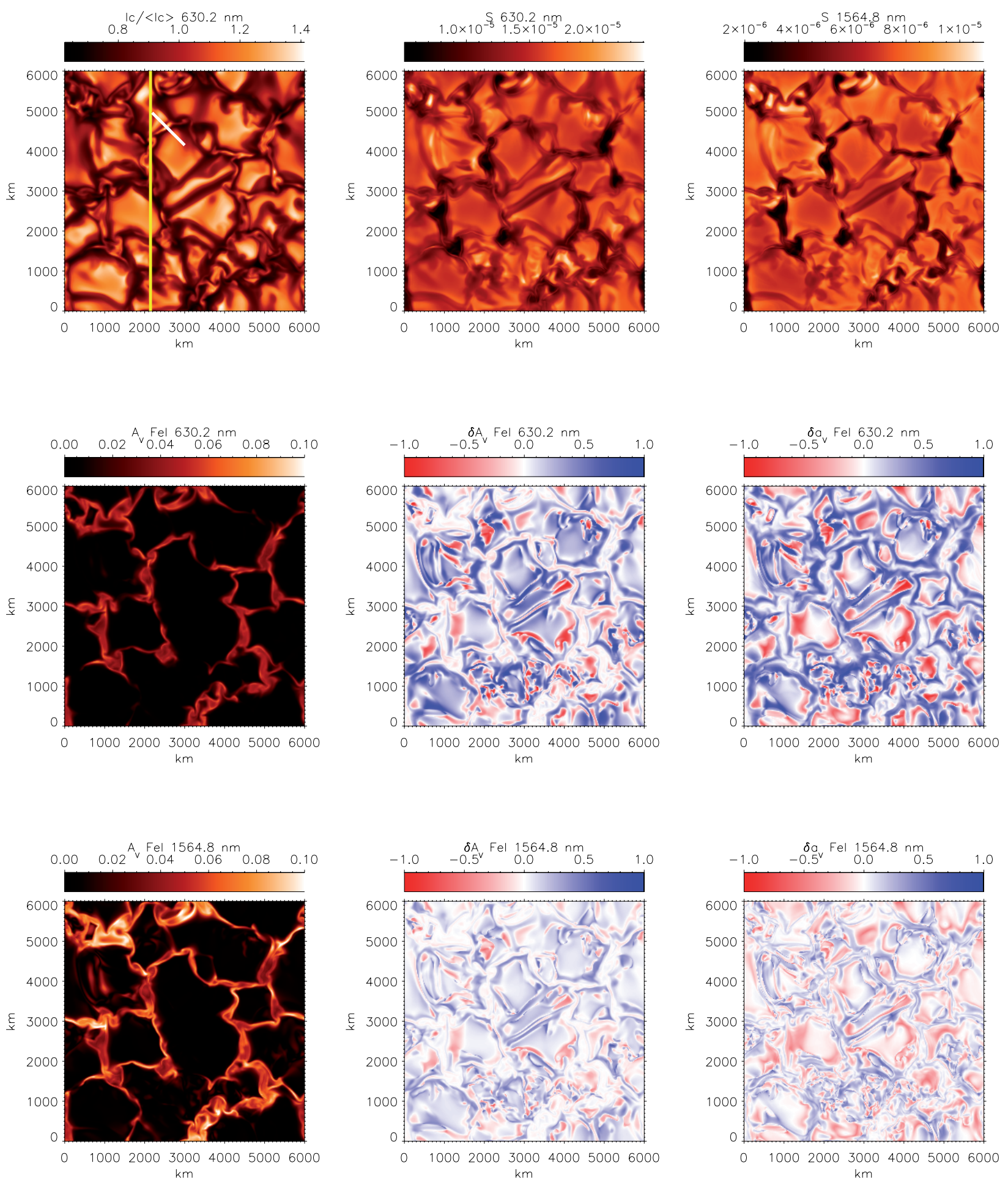

Fig. 3. Properties of synthetic Stokes- $I$ and Stokes- $V$ profiles for the snapshot from the $\left\langle B_{z}\right\rangle=200 \mathrm{G}$ simulation shown in Fig. 1. Given are maps of the (normalized) continuum intensity at $630.2 \mathrm{~nm}$ (top left panel), line strength, $S$ (top middle panel for Fe I $630.2 \mathrm{~nm}$ and top right panel for Fe I $1564.8 \mathrm{~nm}$ ), unsigned Stokes- $V$ area, $A_{V}$ (middle left panel for $630.2 \mathrm{~nm}$ and bottom left panel for $1564.8 \mathrm{~nm}$ ), Stokes- $V$ area asymmetry (central panel for $630.2 \mathrm{~nm}$ and bottom middle panel for $1564.8 \mathrm{~nm}$ ), and Stokes-V amplitude asymmetry (middle right panel for $630.2 \mathrm{~nm}$ and bottom right panel for $1564.8 \mathrm{~nm}$ ). In the continuum intensity image (top left panel), the slit position for Figs. 6 and 5 is indicated by the vertical line and the cut through the magnetic flux sheet shown in Fig. 10 is indicated by the inclined line in the upper left quadrant.

asymmetry. The predominantly positive values indicate that in most cases the "blue" lobe the of Stokes- $V$ profile has larger area and amplitude than the "red" lobe. Lines of sight through the peripheral parts of a flux concentration that expands with height cross its boundary and, in the presence of strong external flows, thus sample significant magnetic field and velocity gradients. As a result, the Stokes- $V$ profiles become asymmetric (Illing et al. 1975) with a stronger blue lobe in the case of dominating external downflows (Grossmann-Doerth et al. 1988b). The asymmetry arises from the fact that the wavelength shifts due to the Doppler effect and the Zeeman splitting have the same sign for one lobe, but opposite sign for the other lobe. 


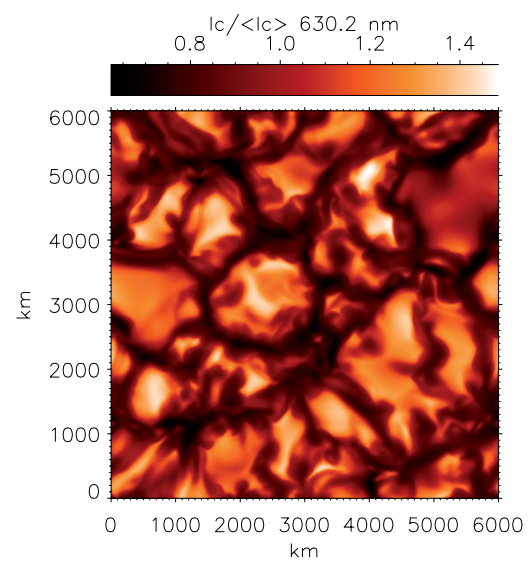

$\mathrm{km}$

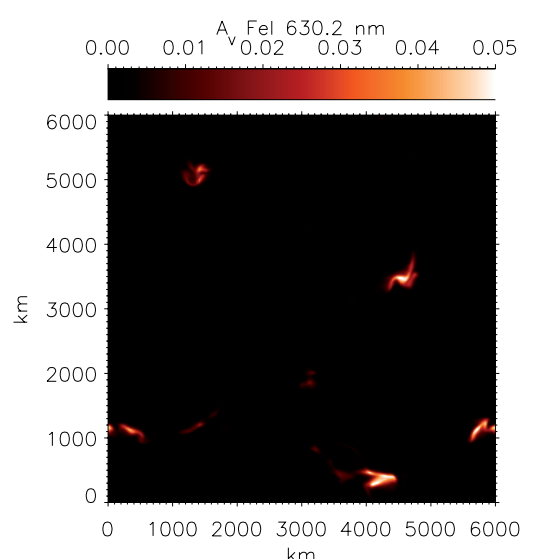

$\mathrm{km}$
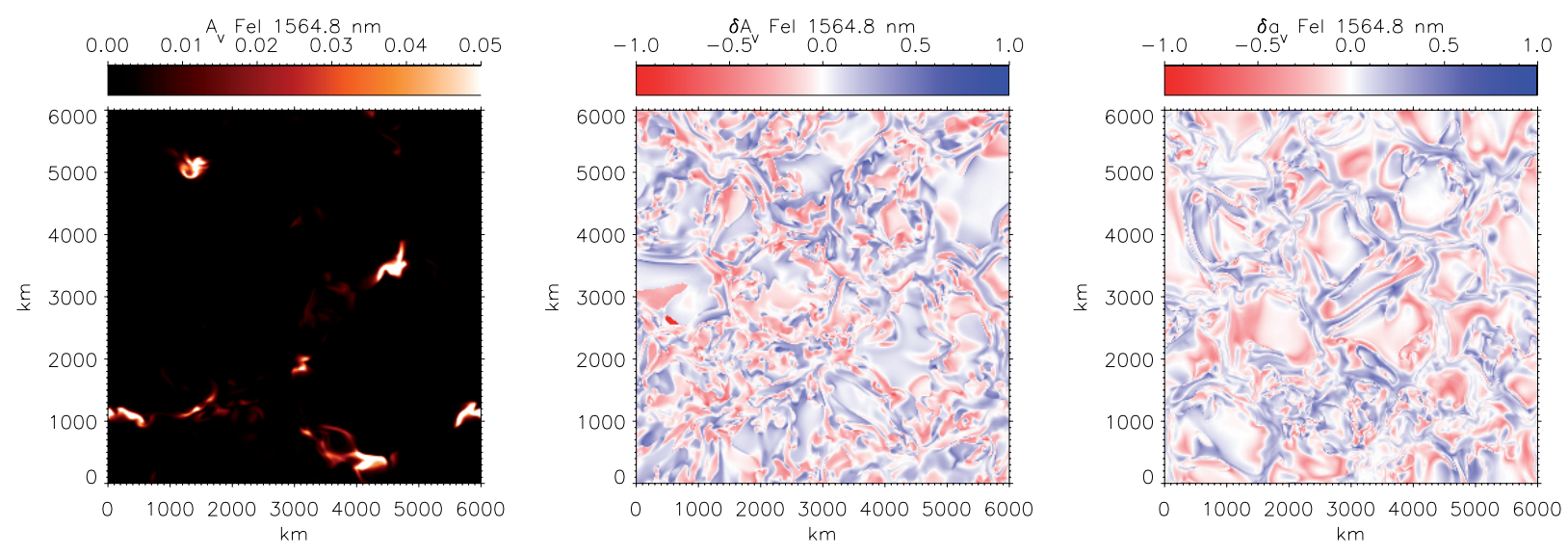

Fig. 4. Same as Fig. 3 for the snapshot from the simulation with $\left\langle B_{z}\right\rangle=10 \mathrm{G}$. The scales are the same as in the previous figure, except for the $A_{V}$ images.

The effect is strongest when both shifts are of similar magnitude; in addition, the area asymmetry depends on line saturation (Grossmann-Doerth et al. 1989; Solanki 1989). This explains why the asymmetries are considerably larger for the visible line, which is stronger (more saturated) and has a smaller Zeeman splitting, better matching the Doppler shift. On the other hand, the spatial distributions of $\delta A_{V}$ and $\delta a_{V}$ from both lines are very similar.

Figure 4 shows the corresponding maps for the $\left\langle B_{z}\right\rangle=$ $10 \mathrm{G}$ run, which could represent an almost decayed unipolar part of an active region or the field underlying a coronal hole. The maps of unsigned Stokes- $V$ area (middle left and bottom left panels) show a significant signal only in a few isolated patches of concentrated magnetic flux. The infrared line displays an enhanced $A_{V}$ not only in the weak-field regions, but also in the stromg flux concentrations. This does not imply that the Stokes- $V$ amplitude is also larger for this line, since the $V$ lobes can become very broad owing to the vertical field gradient (Zayer et al. 1989). The maps of the Stokes- $V$ area and amplitude asymmetries are very complex and show almost no 

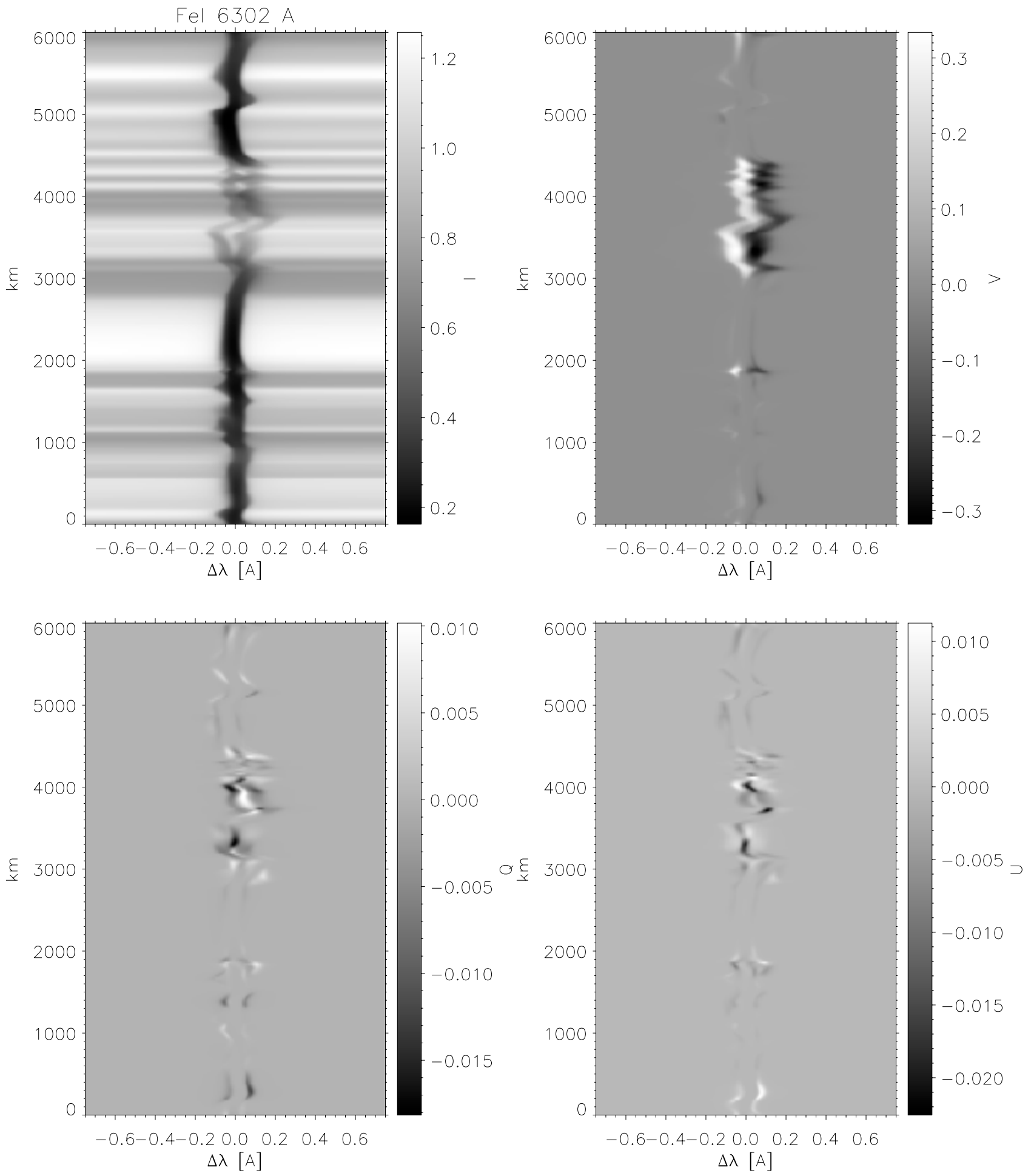

Fig. 5. Synthetic slit spectra, calculated for the $630.2 \mathrm{~nm}$ line. Top left: Stokes-I, top right: Stokes-V/I, bottom left: Stokes- $Q / I$, bottom right: Stokes- $U / I$. The position of the slit is indicated by the vertical line in the top left panel of Fig. 3.

correspondence to the structures in the continuum image. In this case, the strongest asymmetries arise from very weak magnetic fields in granules, with some preference for the granule edges. No sign of the asymmetry parameters is preferred, with the $630.2 \mathrm{~nm}$ showing much larger values.

Synthetic "slit spectra" of all four Stokes parameters along the vertical yellow line on the continuum image for the $200 \mathrm{G}$ snapshot (upper left panel of Fig. 3), are shown in Figs. 5 and 6. The width of the artificial slit is equal to the horizontal cell size of the simulation (about $20 \mathrm{~km}$ on the Sun). The spectra represent "ideal" cases; for a direct comparison with actual observations, one would have to take into account the instrumental profile and finite spatial resolution. The synthetic spectral lines show the characteristic "line wiggles" from the Doppler shifts due to the granular up- and downflows. The Stokes- $V$ spectra (upper right panel) show strong signals where the slit cuts through an extended flux concentration (in the upper half) and through a narrow flux sheet (at $\sim 1800 \mathrm{~km}$ ). The $I$-profiles of 

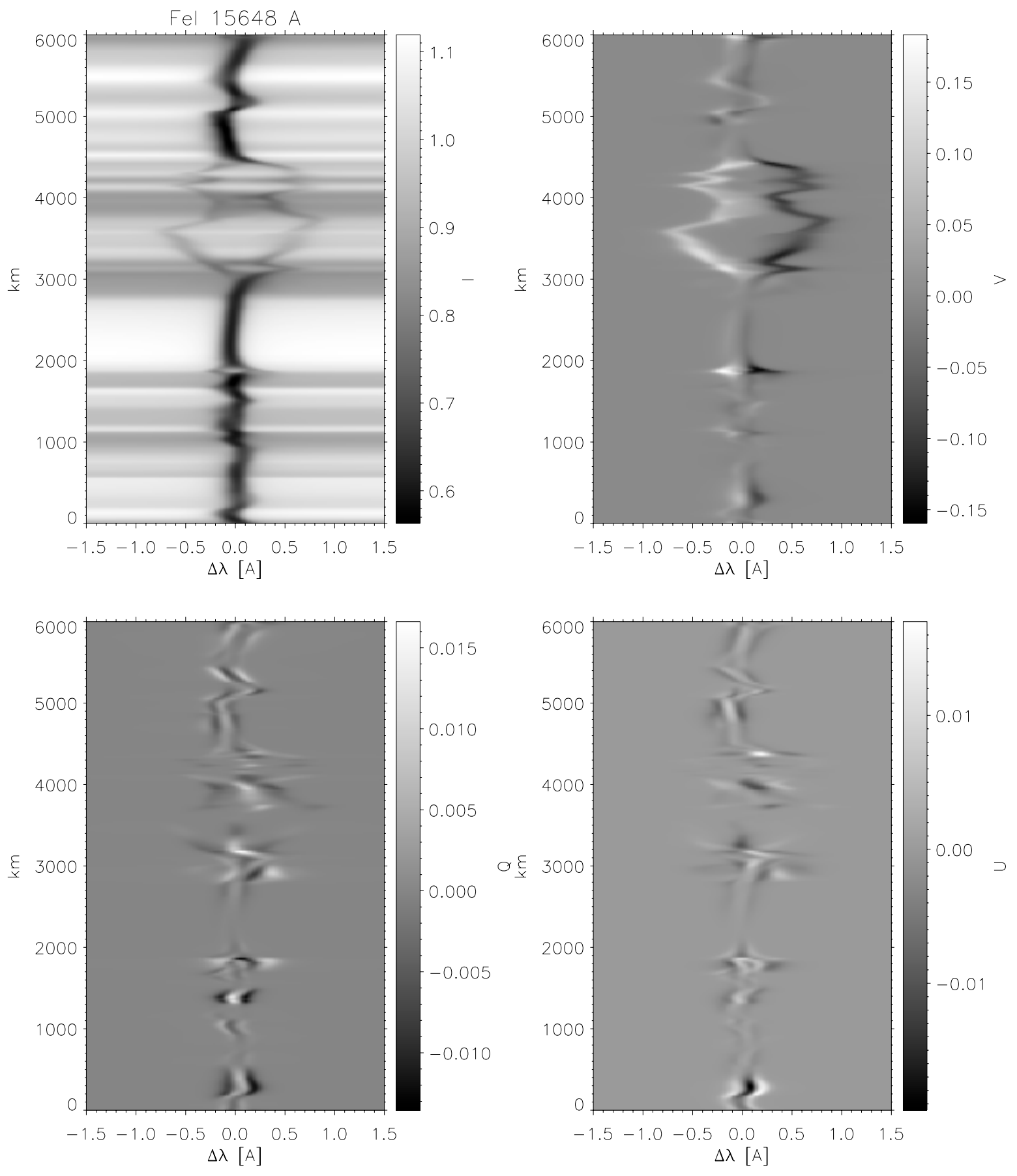

Fig. 6. Same as Fig. 5 for the $1564.8 \mathrm{~nm}$ line.

the lines are weakened and split (more strongly so in the case of the infrared line) at these locations, while strong line wiggles in both Stokes- $I$ and Stokes- $V$ indicate the presence of significant internal structure of the large magnetic flux concentration. Note that some of the variation may be due to the fact that the large, elongated flux sheet is bent, so that central and peripheral regions are sampled at different locations along the slit. The spectra of Stokes- $Q$ and $U$ show rather weak signals, consistent with the lack of significant horizontal magnetic field components in the strong flux concentrations. As expected from its larger Zeeman sensitivity, the infrared line displays larger values of $Q$ and $U$.

In order to compare spectroscopically determined velocities with their actual values in the simulation, we define the

line-of-sight velocity, $\Delta v$, according to the Doppler shift of the Stokes-I profile, viz.

$\Delta v=\frac{c\left(\lambda_{0}-\lambda_{\mathrm{cg}}\right)}{\lambda_{0}}$

where $c$ is the velocity of light, $\lambda_{0}$ is the wavelength of the line center in the rest frame, and $\lambda_{\text {cg }}$ is the wavelength corresponding to the center of gravity of the line. The latter is defined as the first moment of the profile,

$\lambda_{\mathrm{cg}}=\frac{\int\left(I_{\mathrm{c}}-I\right) \lambda \mathrm{d} \lambda}{\int\left(I_{\mathrm{c}}-I\right) \mathrm{d} \lambda}$,

where $I_{\mathrm{c}}$ is the continuum intensity and $I$ is the line intensity. 

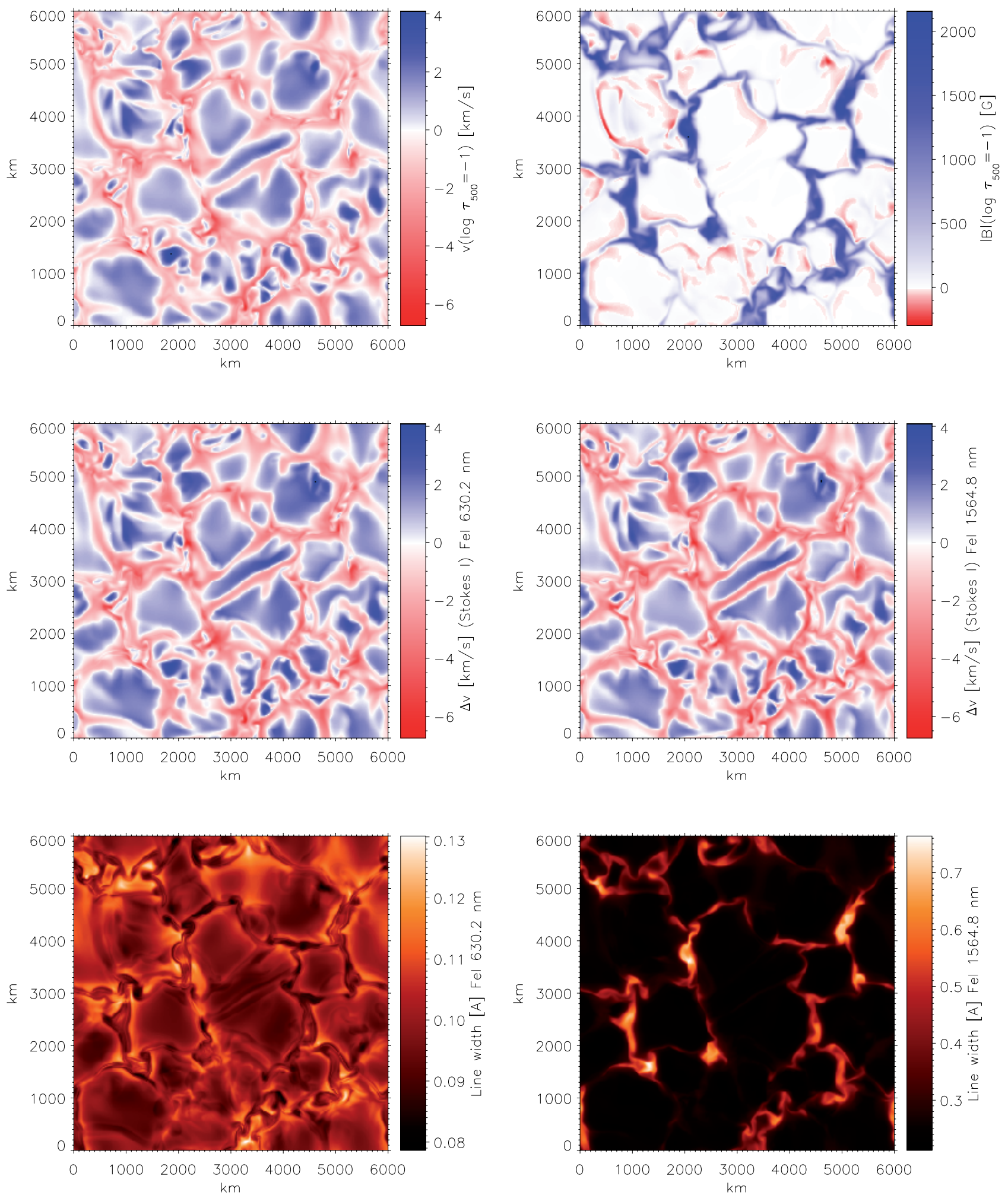

Fig. 7. Vertical velocity (top left panel) and field strength (top right panel) at the surface $\tau_{500}=0.1$ for the $\left\langle B_{z}\right\rangle=200 \mathrm{G}$ simulation. The middle row shows maps of the vertical velocity as determined from the Doppler shift of the $630.2 \mathrm{~nm}$ line (middle left panel) and the $1564.8 \mathrm{~nm}$ line (middle right panel). The bottom row gives maps of the line width of the $630.2 \mathrm{~nm}$ line (bottom left panel) and the $1564.8 \mathrm{~nm}$ line (bottom right panel).

Figure 7 (for the $\left\langle B_{z}\right\rangle=200 \mathrm{G}$ simulation) and Fig. 8 (for $\left.\left\langle B_{z}\right\rangle=10 \mathrm{G}\right)$ give a comparison between the velocity $\Delta v$ determined from the Doppler shift of the $630.2 \mathrm{~nm}$ (middle left panel) and $1564.8 \mathrm{~nm}$ lines (middle right panel), respectively, with the actual flow pattern of the simulation at the surface $\tau_{500}=0.1$ (top left panel). This level can be seen as a very rough representation of the optical depth range contributing to the spectral line shift. The maps show that the actual velocities at that height and the spectroscopically determined velocities qualitatively agree. The somewhat larger flow velocities 

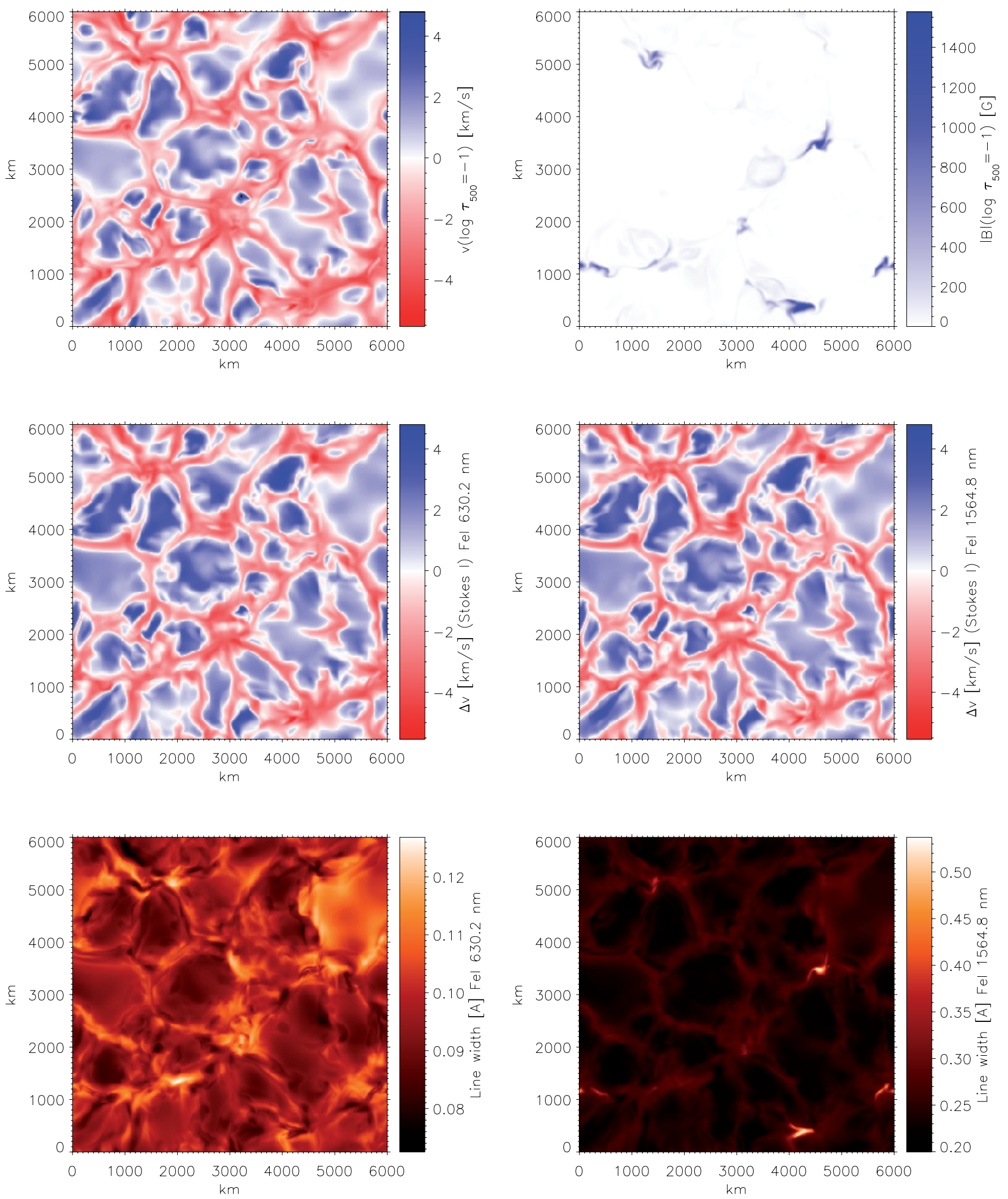

Fig. 8. Same as Fig. 7 for the $\left\langle B_{z}\right\rangle=10 \mathrm{G}$ simulation.

returned by the infrared line are consistent with its lower height of formation since the flow speeds tend to increase with depth. However, the corresponding scatter plots given in Fig. 9 show that the scatter of the individual points is considerable. This is not surprising in view of the extended height range of line formation (or better, the finite width of the velocity response function in combination with significant vertical velocity gradients). In fact, it has been shown that a much better representation of the height to which the center-of-gravity shift of Stokes- $I$ corresponds can be determined via response functions (Beckers \& Milkey 1975; Landi Degl'Innocenti \& Landi Degl'Iinnocenti 1977; Grossmann-Doerth et al. 1988a; Del Toro Iniesta et al. 1994; Sanchez Almeida et al. 1996; Cabrera Solana et al. 2005; Del Toro Iniesta 2003, Sect. 10.4.3). Nevertheless, it is interesting to see that, for a first guess, the level $\tau_{500}=0.1$ is not a completely unreasonable choice. 

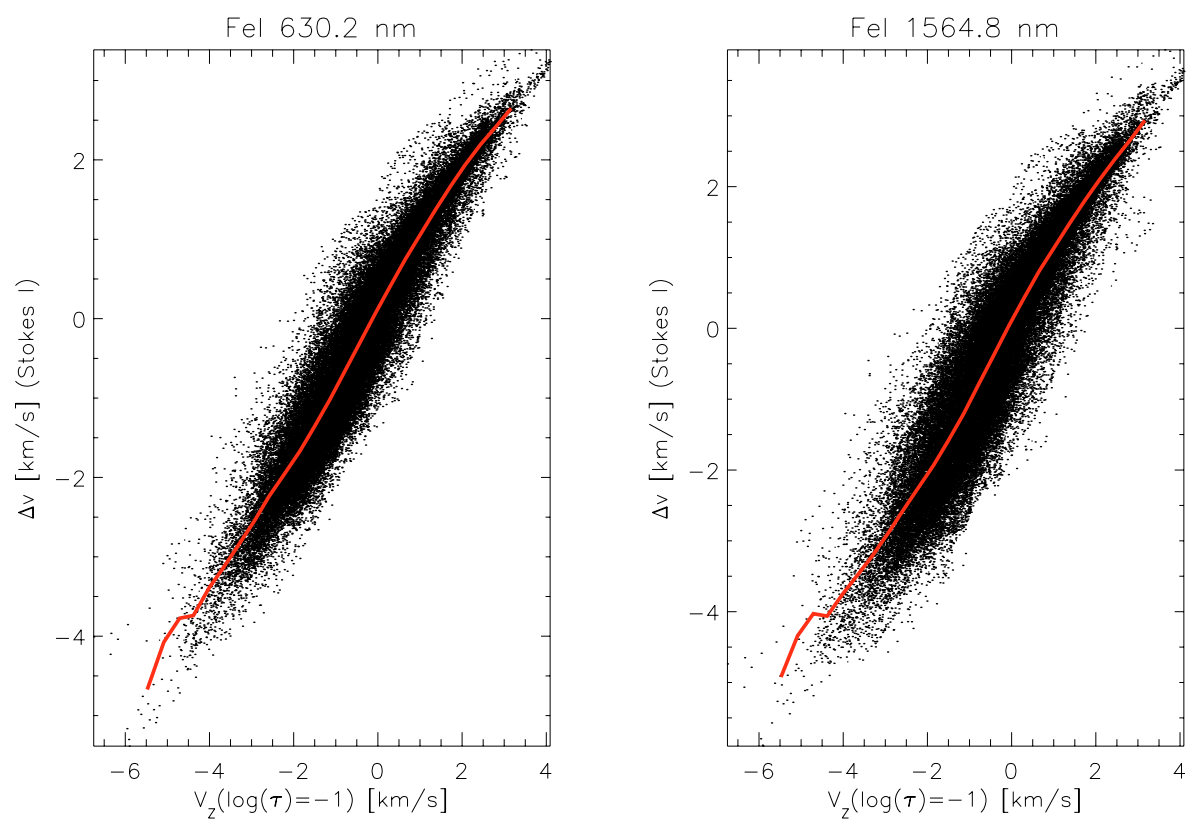

Fig. 9. Scatter plots of velocity determined from the Doppler shift of the center of gravity of the spectral line versus the vertical velocity at $\tau_{500}=0.1$ from the simulation $(200 \mathrm{G})$. The line shows the binned average.
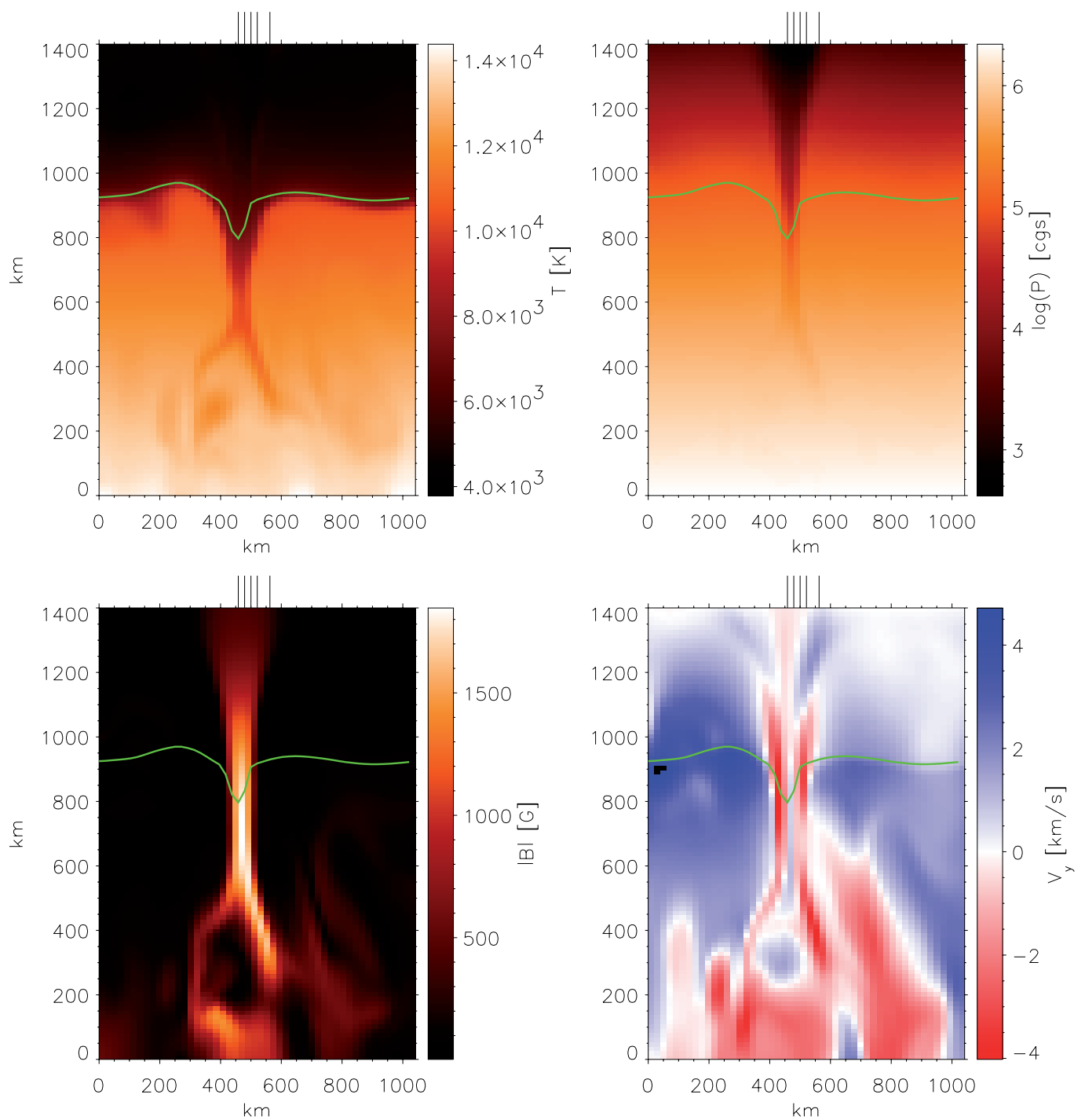

Fig. 10. Temperature (upper left panel), gas pressure (upper right panel), magnetic field strength (lower left panel), and vertical velocity (lower right panel) for the vertical cut through the simulation box marked by the white line on the upper left panel of Fig. 3 . The green curve indicates the level $\tau_{500}=1$. Negative values of the velocity (shown in red) correspond to downflows. The lines of sight corresponding to the Stokes profiles and gradients shown in Fig. 11 are indicated at the top of each panel. 
Fel $1564.8 \mathrm{~nm}$
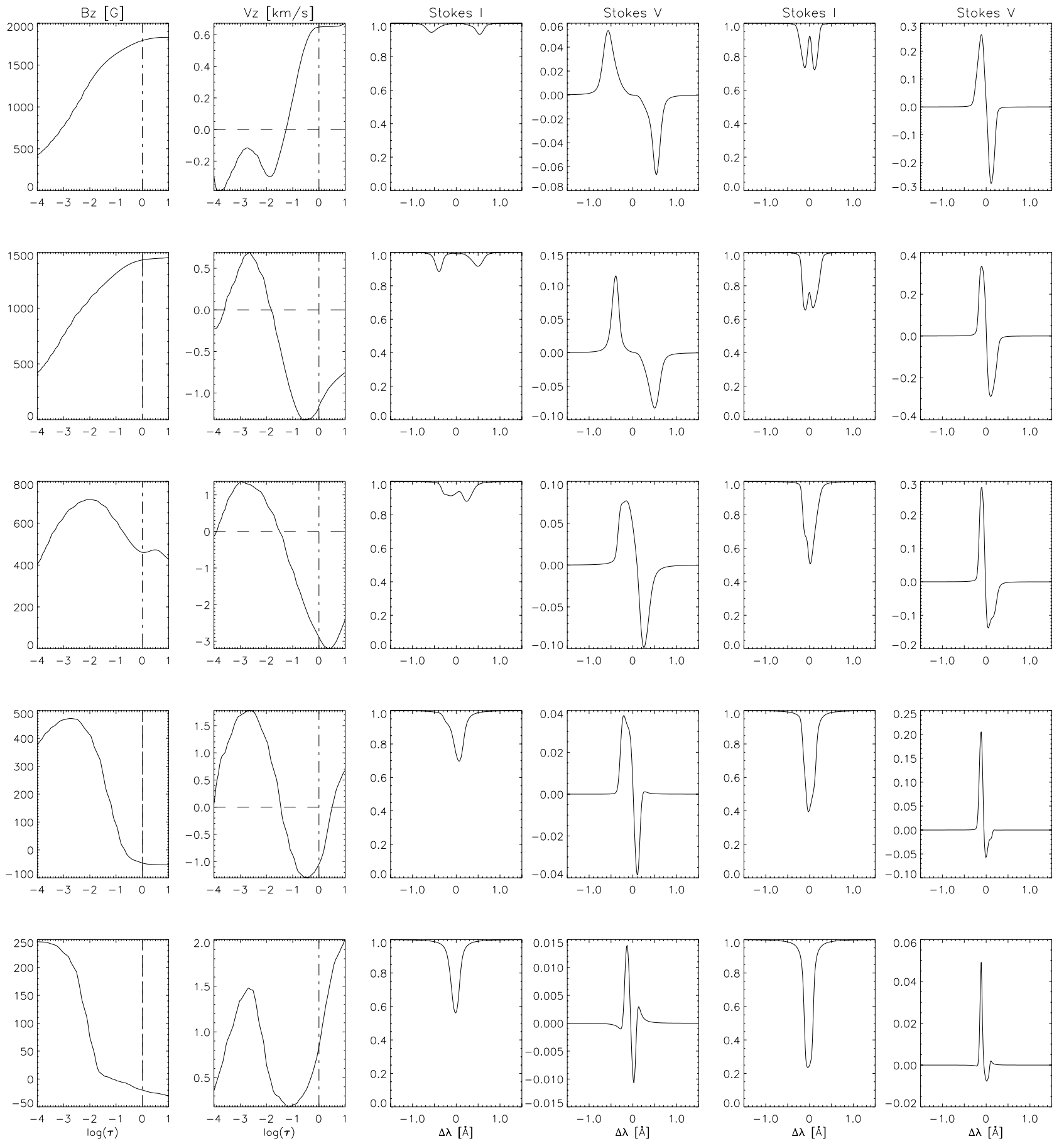

Fig. 11. Stokes- $I$ and Stokes- $V$ profiles for the $1564.8 \mathrm{~nm}$ line (third and fourth column) and for the $630.2 \mathrm{~nm}$ line (fifth and sixth column). The corresponding magnetic field and velocity profiles as a function of $\log \tau_{500}$ are given in the first two columns. The level $\tau_{500}=1$ is marked by the dash-dotted line; zero vertical velocity is indicated by the dashed line. Each row corresponds to one of the vertical lines of sight indicated in Fig. 10, ranging from the central part of the flux concentration (top row) to its outer periphery (bottom row).

The bottom panels of Figs. 7 and 8 show maps of the line width, $\Delta \lambda$, which is defined here as the standard deviation of the profile (square root of the second moment):

$\Delta \lambda=\sqrt{\frac{\int\left(I_{\mathrm{c}}-I\right)\left(\lambda-\lambda_{\mathrm{c}}\right)^{2} \mathrm{~d} \lambda}{\int\left(I_{\mathrm{c}}-I\right) \mathrm{d} \lambda}}$.

The maps of $\Delta \lambda$ (bottom left panel for $630.2 \mathrm{~nm}$, bottom right panel for $1564.8 \mathrm{~nm}$ ) reflect the different magnetic sensitivity of the visible and infrared Fe I lines. In the case of $630.2 \mathrm{~nm}$, the line width is actually decreased in the bulk of the magnetic flux concentrations, showing that its Zeeman sensitivity is insufficient for a significant broadening. On the other hand, there is a clear increase of $\Delta \lambda$ in the periphery of the flux concentrations, where the field is weaker but strong downflows prevail. In connection with the fact that the line width is found to be also enhanced in non-magnetic intergranular downflow lanes (see Fig. 8), this indicates that the strong velocity gradients in 


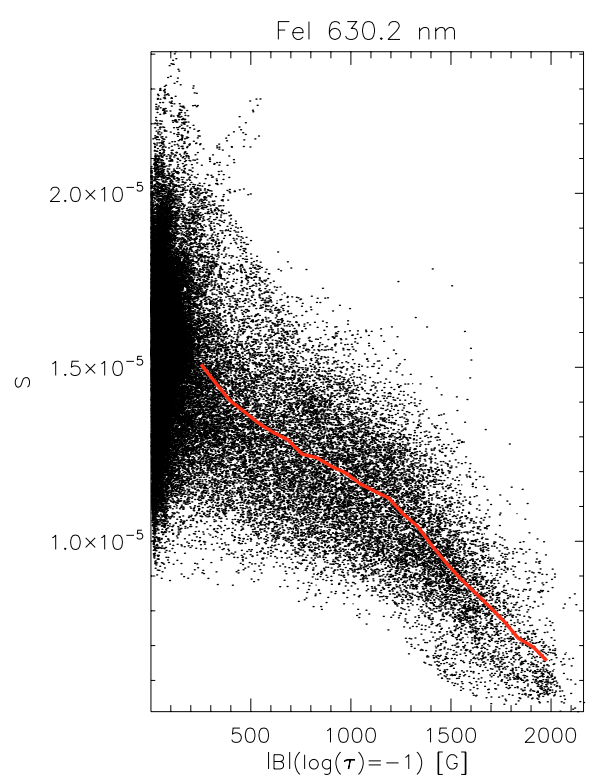

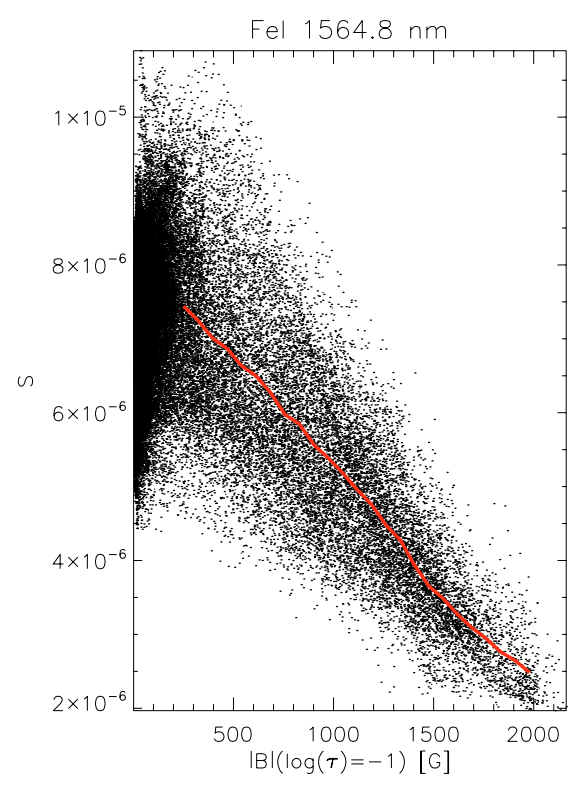

Fig. 12. Scatter plots of line strength versus magnetic field strength at $\tau_{500}=0.1$ for the snapshot from the $\left\langle B_{z}\right\rangle=200 \mathrm{G}$ simulation. a) Fe I $630.2 \mathrm{~nm}$, b) Fe I $1564.8 \mathrm{~nm}$. The lines show the binned averages. Note the different vertical scales on the two panels.
Table 2. Stokes- $V$ amplitude asymmetry $\left(\delta a_{V}\right)$ and area asymmetry $\left(\delta A_{V}\right)$ for the $630.2 \mathrm{~nm}$ and $1564.8 \mathrm{~nm}$ lines along 5 lines of sight (l.o.s.) in Figs. 10 and 11. The 1.o.s. \# 1 is near the center of the flux sheet while l.o.s. \# 5 is in its far periphery.

\begin{tabular}{crrrr}
\hline \hline & \multicolumn{2}{c}{$630.2 \mathrm{~nm}$} & \multicolumn{2}{c}{$1564.8 \mathrm{~nm}$} \\
\hline 1.o.s. \# & $\delta a_{V}$ & $\delta A_{V}$ & $\delta a$ & $\delta A$ \\
\hline 1 & -0.02 & 0.03 & -0.11 & 0.01 \\
2 & 0.06 & -0.07 & 0.16 & -0.03 \\
3 & 0.31 & 0.05 & -0.12 & 0.04 \\
4 & 0.51 & 0.43 & -0.02 & 0.33 \\
5 & 0.72 & 0.59 & 0.13 & 0.27 \\
\hline
\end{tabular}

downflows are responsible for the broadening of the visible line (Nesis et al. 1996; Solanki et al. 1996). In the case of the more Zeeman-sensitive $1564.8 \mathrm{~nm}$ infrared line, the maps show a clear correspondence between the magnetic field strength and the line width. Doppler broadening is relevant for this line as well, since some enhancement of the line width can be seen in all downflow lanes, even in the absence of a strong field.

\subsection{Cut through a magnetic feature}

A two-dimensional vertical cut through a sheet-like magnetic feature along the inclined white line in the upper left panel of Fig. 3 is shown in Fig. 10. The four panels display the distributions of temperature (top left), gas pressure (top right), magnetic field strength (bottom left), and vertical velocity (bottom right). The green line on the images indicates the $\tau_{500}=1$ level. The analyzed flux sheet is rather shallow, the flux concentration extending only to a depth of about $500 \mathrm{~km}$ below the average level of $\tau_{500}=1$. With respect to its surroundings at the same geometrical depth, the gas in the interior of the magnetic flux sheet is cooler and has a deficit in gas pressure, which is almost completely compensated by the magnetic pressure, in accordance with the approximation of thin flux tubes (Shelyag 2004). There are strong downflows with velocities up to $3 \mathrm{~km} \mathrm{~s}^{-1}$ at the periphery of the magnetic flux sheet, while the flow speeds in its interior are smaller, exhibiting an upflow of up to $600 \mathrm{~m} \mathrm{~s}^{-1}$ near $\tau_{500}=1$. Note that the velocity plot in Fig. 10 shows only the vertical component: since the flow is largely subsonic, a

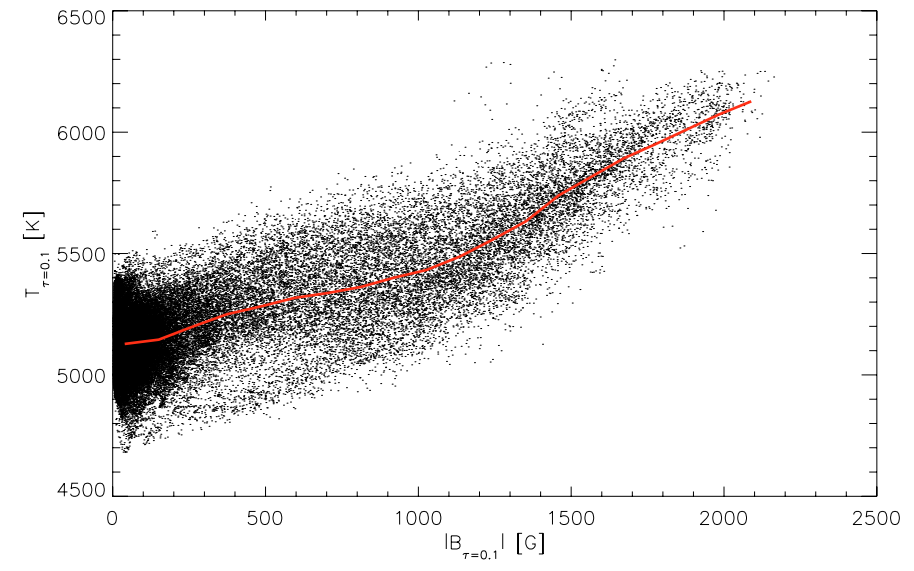

Fig. 13. Scatter plots of temperature versus magnetic field strength (both at $\tau_{500}=0.1$. The line shows the binned average.

divergence of the vertical mass flux at its stagnation points is balanced by a convergence of the horizontal mass flux, and vice versa. The height level of $\tau_{500}=1$ is depressed by about $150 \mathrm{~km}$ in the region of strong magnetic field. This is caused by the lower gas temperature and density in the flux sheet, an effect analogous to the Wilson depression in sunspots.

Figure 11 shows the profiles of magnetic field and velocity along several vertical lines of sight (indicated by the short vertical lines at the top of the panels in Fig. 10) together with the emergent Stokes- $I$ and Stokes- $V$ profiles at these locations. Each line of sight corresponds to one row of panels in Fig. 11, which show (from left to right) the line-of-sight components of the magnetic field and the velocity field as a function of $\tau_{500}$, and the Stokes- $I$ and $V$ profiles of the $1564.8 \mathrm{~nm}$ and $630.2 \mathrm{~nm}$ lines. The first row in Fig. 11 represents the center of the flux sheet, showing a slow upflow and large field strength in the height range relevant for line formation, so that the Stokes profiles are split and nearly symmetric. The field strength drops with height in accordance with the horizontal pressure balance. Moving towards the periphery of the flux concentration, the lines of sight cut through the magnetopause of the expanding magnetic structure into the surrounding downflow (cf. the last three rows of Fig. 11, which show an increase of the field strength with height 

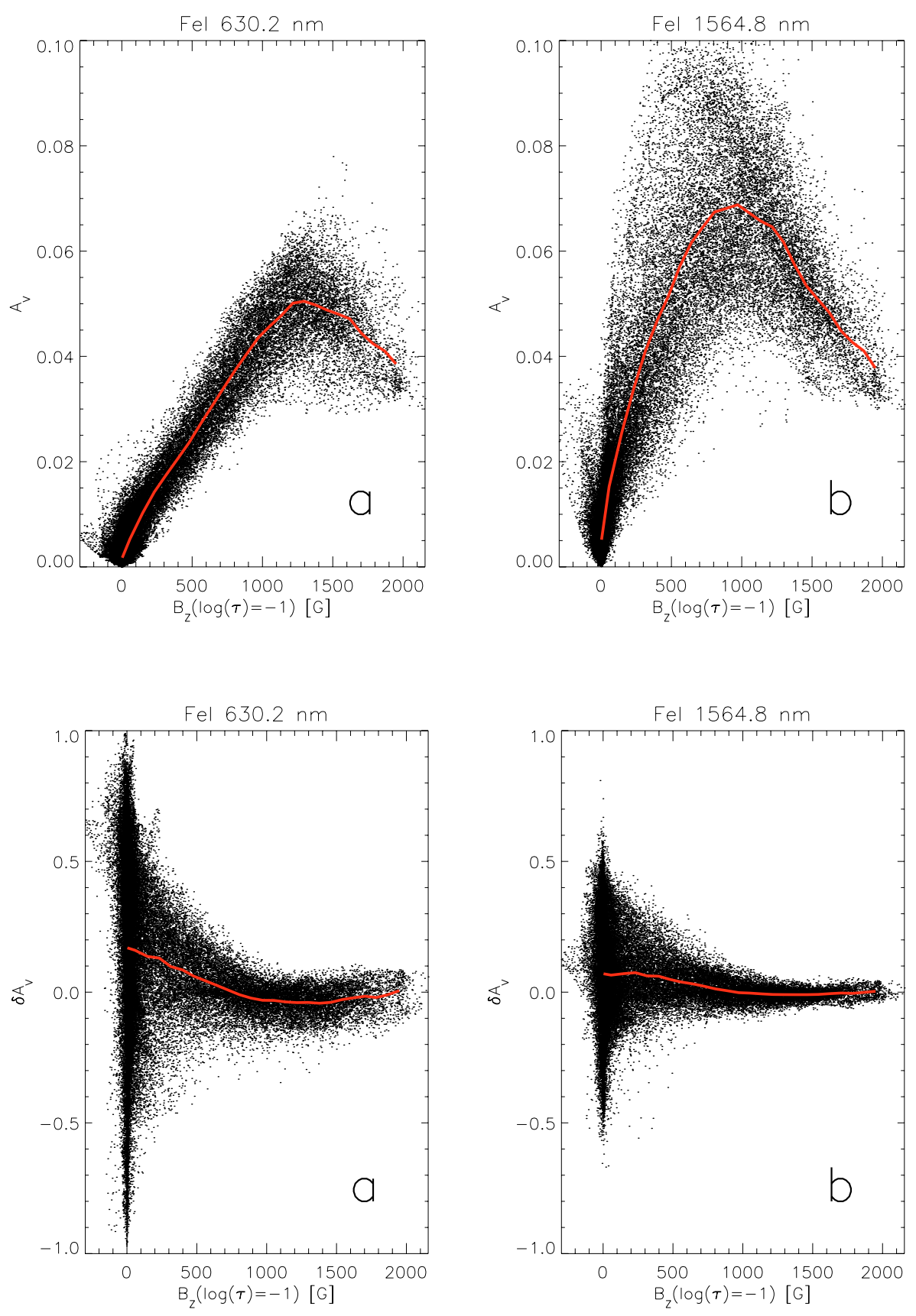

Fig. 14. Scatter plots of the unsigned Stokes$V$ area versus the vertical (line-of-sight) component of the magnetic field at $\tau_{500}=0.1$ a) Fe I $630.2 \mathrm{~nm}$, b) Fe I $1564.8 \mathrm{~nm}$. The lines show binned averages.

Fig. 15. Scatter plots of the Stokes- $V$ area asymmetry versus the vertical (line-of-sight) component of the magnetic field at $\tau_{500}=0.1$ a) Fe I $630.2 \mathrm{~nm}$, b) Fe I $1564.8 \mathrm{~nm}$. The lines show binned averages.

in the lower photosphere). As a result, the Zeeman splitting is smaller and the $V$-profiles, particularly for the visible line, become strongly asymmetric.

Illing et al. (1975) have shown that asymmetric Stokes- $V$ profiles arise in the presence of magnetic field and velocity gradients along the line of sight, the sign of the asymmetry being determined by the sign of the product of both gradients. On this basis, a mechanism leading to strongly asymmetric (and unshifted) Stokes- $V$ profiles in the periphery of a static magnetic flux concentration has been suggested by Grossmann-Doerth et al. (1988c) and Solanki (1989). These authors assumed an idealized two-component atmosphere model: the upper part of the atmosphere has a magnetic field and is static while the lower part is non-magnetic and exhibits a systematic vertical flow. The Stokes- $V$ profile formed along a vertical line of sight that cuts through both components then becomes asymmetric with an unshifted zero-crossing wavelength. Solanki \& Pahlke (1988) showed that the blue wing of the Stokes- $V$ profile is stronger than the red wing (positive area asymmetry) if the inequality

$$
\frac{\mathrm{d}\left|B_{\mathrm{los}}\right|}{\mathrm{d} \tau} \cdot \frac{\mathrm{d} v_{\mathrm{los}}}{\mathrm{d} \tau}>0
$$

holds, where $B_{\text {los }}$ and $v_{\text {los }}$ are the line-of-sight components of the magnetic field and the flow velocity, respectively. Note that positive values of the velocity correspond to upflows. The asymmetry is positive in regions where the velocity and the magnetic field gradients have the same sign, such as in the periphery of the flux sheet shown in Figs. 10 and 11. The area asymmetries of the $V$-profiles corresponding to the five lines of sight considered in Fig. 11 are in agreement with this rule (compare Table 2 with the first two columns of Fig. 11). Note that the Stokes- $V$ profiles are formed higher in the atmosphere near the periphery of the 

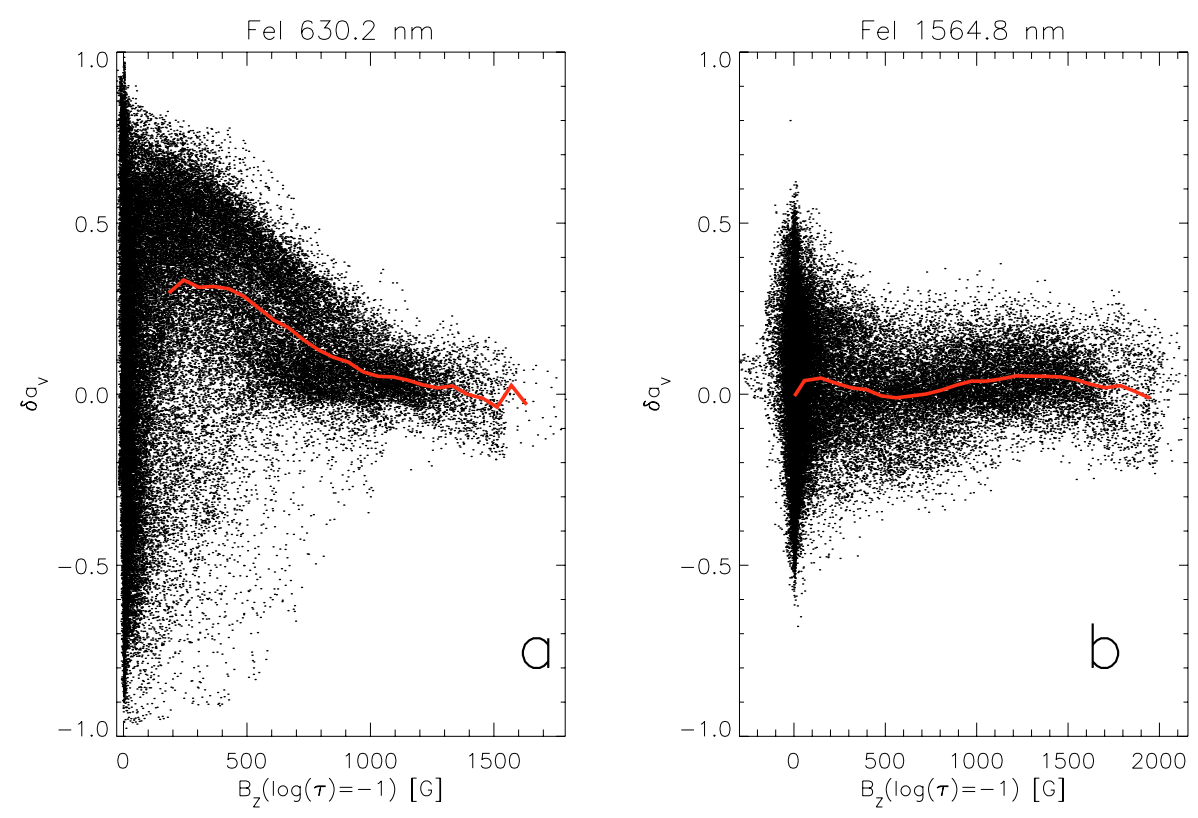

Fig. 16. Same as Fig. 15 for the Stokes- $V$ amplitude asymmetry.
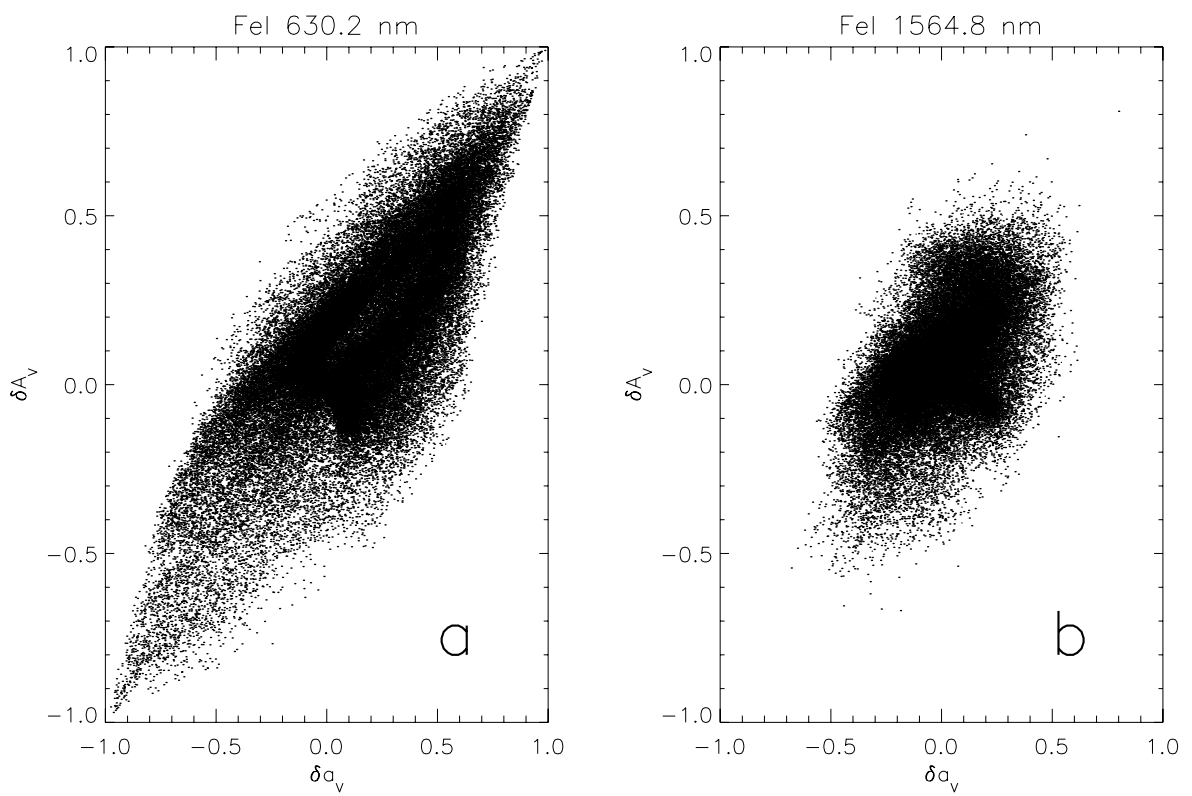

Fig. 17. Scatter plots of Stokes- $V$ area asymmetry versus Stokes- $V$ amplitude asymmetry. a) Fe I $630.2 \mathrm{~nm}$, b) Fe I $1564.8 \mathrm{~nm}$.

flux tube, since the they obtain a larger contribution from layers with a stronger field. The fact that the $630.2 \mathrm{~nm}$ line generally shows larger $V$-profile asymmetry than $1564.8 \mathrm{~nm}$ line is due to the combination of stronger saturation and weaker splitting of the visible line (Grossmann-Doerth et al. 1989; Solanki 1989).

\subsection{Statistical properties}

We now consider the complete set of Stokes profiles calculated for vertical lines of sight through each of the $288^{2}$ horizontal resolution elements of $20.8 \times 20.8 \mathrm{~km}^{2}$ size each, which are provided by a simulation snapshot. We study the dependence of their properties on the magnetic field and flow structure using the snapshot from the $\left\langle B_{z}\right\rangle=200 \mathrm{G}$ simulation.

Scatter plots of the line strength $S$ versus magnetic field strength at $\tau_{500}=0.1$ for the $630.2 \mathrm{~nm}$ line (Fig. 12a) and for the $1564.8 \mathrm{~nm}$ line (Fig. 12b) show that both lines are progressively weakened with increasing magnetic field strength. The binned averages given by the line indicate an almost linear dependence, in the case of the $630.2 \mathrm{~nm}$ line with some increase of the slope for large field strength. The scatter plot of temperature versus field strength (both at $\tau_{500}=0.1$ ) shown in Fig. 13 indicates that the line weakening is largely due to the larger temperature (at equal optical depth) in the magnetic flux concentrations (cf. Chapman \& Sheeley 1968), resulting from the lateral radiative heating and reduced density of these structures. However, the visible line, which should be more temperature sensitive owing to its lower value of $\chi_{\mathrm{e}}$, shows a somewhat lower relative weakening than the infrared line. This indicates that other effects, such as Zeeman desaturation as well as vertical velocity and temperature gradients, also affect the line strength in a significant way. A more detailed consideration on the basis of response functions (e.g., Del Toro Iniesta 2003, Sect. 10.4.2) is required to shed more light on this result, but such an analysis is beyond the scope of this paper.

Figure 14 shows scatter plots of the unsigned Stokes- $V$ area, $A_{V}$ versus the strength of the vertical (line-of-sight) component of magnetic field at $\tau_{500}=0.1$. It illustrates the different 


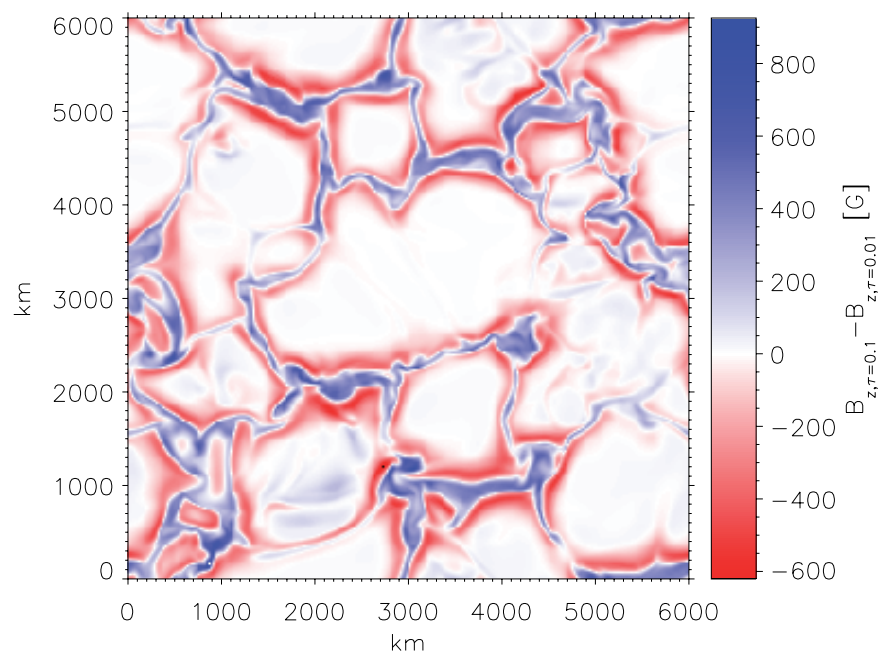

Fig. 18. Spatial distribution of $\left(B_{z, \tau_{500}=0.1}-B_{z, \tau_{500}=0.01}\right)$, representing the average line-of-sight gradient of the vertical magnetic field. Magnetic field increasing with optical depth is indicated by blue color, magnetic field decreasing with optical depth is shown in red.

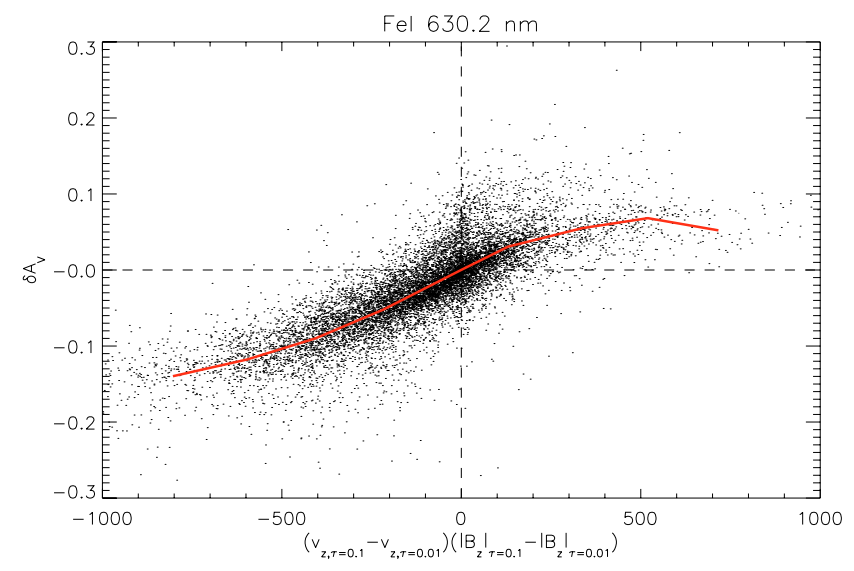

Fig. 19. Scatter plot of the Stokes- $V$ area asymmetry for the $630.2 \mathrm{~nm}$ line versus a quantity representing the gradients of the line-of-sight magnetic field and velocity within the height range of line formation. The sign of the bin-averaged $\delta A_{V}$ (indicated by the line) is in agreement with the relation given in Eq. (8).

magnetic sensitivity of the two lines. The Stokes signal of the $630.2 \mathrm{~nm}$ line depends linearly on the magnetic field strength until the Zeeman splitting is no longer small compared to the line width and saturation sets in for $B_{z} \gtrsim 1200 \mathrm{G}$. The curve for the infrared line with its higher Zeeman sensitivity becomes nonlinear at lower field strength and bends over around $900 \mathrm{G}$. This line also exhibits a larger scatter of $A_{V}$ values for intermediate field strengths. The decrease of the Stokes- $V$ area of both lines for large field strength is due to the temperature-induced line weakening in the strong flux concentrations (cf. Fig. 12). The more rapid decrease of $A_{V}$ with $B_{z}$ for the the $1564.8 \mathrm{~nm}$ line is consistent with the stronger weakening of this line as shown in Fig. 12b.

Figures 15 and 16 show scatter plots of the Stokes- $V$ area asymmetry, $\delta A_{V}$, and amplitude asymetry, $\delta a_{V}$, respectively, versus the vertical (line-of-sight) magnetic field strength. The binned averages of the asymmetries given by the red curves show that the asymmetries decrease towards stronger fields, even with a tendency to become negative. The results for the $630.25 \mathrm{~nm}$ line are in qualitative agreement with the observations of
Martínez Pillet et al. (1997, see their Figs. 15 and 16) and Sigwarth et al. (1999, see their Fig. 6). Note, however, the difference in spatial resolution between simulation and observations, so that a more quantitative comparison is not indicated.

The asymmetry distributions shown here are in qualitative agreement with the results of Khomenko et al. (2005b) on the basis of a MURaM simulation of a decaying mixed-polarity field. In both cases (and in agreement with observations) it is found that the amplitude asymmetry, on average, is a factor 2 to 3 larger than the area asymmetry. On the basis of calculations with an idealized flux tube model, Bellot Rubio et al. (1997) have suggested that a downflow in the flux concentration, in addition to the external downflow, could lead to asymmetries with this property. In fact, the simulated flux concentrations, on average, show internal downflows (see Fig. 20), consistent with this suggestion.

The asymmetries for the $1564.8 \mathrm{~nm}$ line are systematically smaller than those for the visible line. The asymmetry is maximized if the wavelength shift due to the Zeeman splitting is similar to the Doppler shift due to the velocity gradient (Grossmann-Doerth et al. 1988b). This is roughly the case for the visible line while the stronger Zeeman sensitivity of the infrared line leads to smaller asymmetry compared to that of the visible line. Furthermore, the infrared line is weaker and less saturated than the visible line, which additionally reduces the asymmetry (Solanki 1989). The large scatter of the asymmetry values for magnetic field strengths below $\sim 200 \mathrm{G}$ is consistent with the observed asymmetries of weak Stokes- $V$ profiles (e.g., Sigwarth 2001) and indicates that the scatter in such observations is not entirely due to noise. In the simulations, many of the weak $V$ profiles result from strongly inclined fields in the granular upflows. Note that, according to the definitions of the asymmetries given by Eqs. (3) and (4), complex profiles, e.g. those with 3 or more lobes, cannot be distinguished from normal profiles in Figs. 15 and 16. Figure 17 shows that the average amplitude and area asymmetries are well correlated, particularly for the $630.2 \mathrm{~nm}$ line, which is in accordance with the observational results of Sigwarth et al. (1999, see their Fig. 9).

Considering the average $V$-profile of the $630.25 \mathrm{~nm}$ line over the whole computational domain of $6 \mathrm{Mm} \times 6 \mathrm{Mm}$, we find values of $\delta A_{V}=3.6 \%$ for the area asymmetry and $\delta a_{V}=17.8 \%$ for the amplitude asymmetry. This can be compared with similar averages (albeit with different averaging areas) of observed plage spectra by Martínez Pillet et al. (1997, see their Fig. 14), who find $\delta A_{V}=3.5 \ldots 4.5 \%$ and $\delta a_{V}=9 . .11 \%$ and by Sigwarth et al. (1999, see their Fig. 13), who give values of $\delta A_{V}=3 \%$ and $\delta a_{V}=11 \%$. The dominance of positive asymmetries (i.e., stronger blue wing of the Stokes- $V$ profile) for intermediate field strengths in Figs. 15 and 16 supports the interpretation that these average asymmetries originate in the peripheral parts of magnetic flux concentrations, which expand with height and are surrounded by strong downflows (such as the example shown in Figs. 10 and 11; see also Table 1). Lines of sight in the periphery of a flux concentration cut through the magnetopause and thus exhibit a reversal of gradient of the line-of-sight magnetic field (field strength decreasing with depth) in comparison to the central parts of the flux concentrations, where the field strength increases with depth (see Fig. 18). Together with the increase of the (downflow) velocity with depth, this leads to positive asymmetries of the Stokes- $V$ profiles originating in the peripheral parts of the flux concentrations (see the central frame of Fig. 3. According to Eq. (8), the sign of the area asymmetry should depend on the product of the gradients of magnetic field and velocity along the line of sight. Figure 19 shows the dependence of the Stokes- $V$ area asymmetry for the $603.2 \mathrm{~nm}$ line on the 


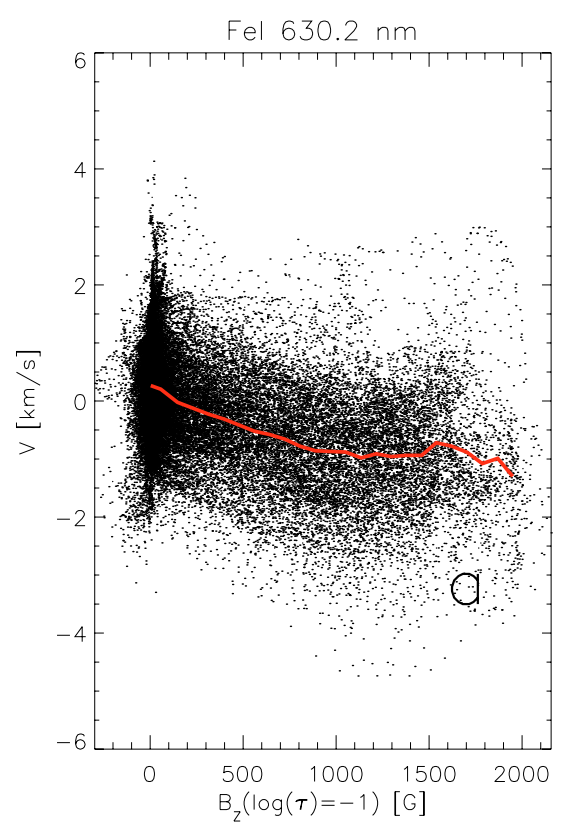

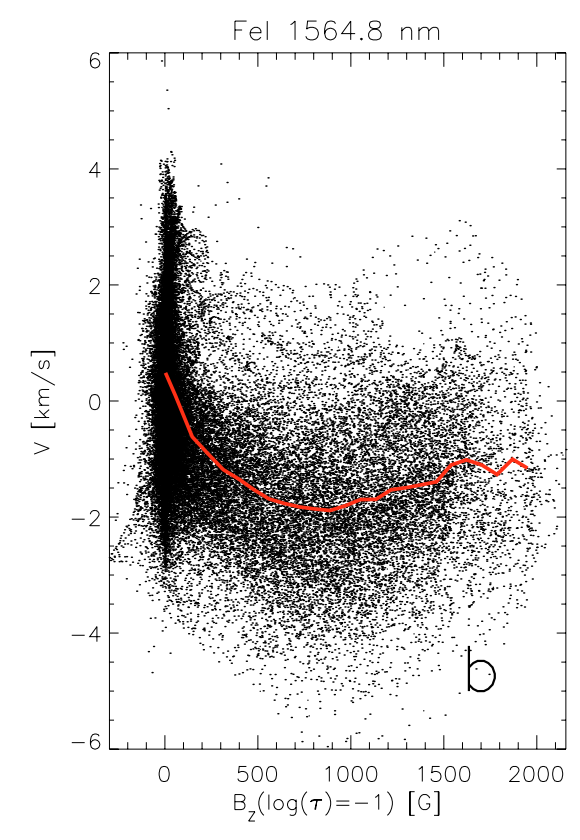

Fig. 20. Scatter plots of the line-of-sight velocity calculated from the zero-crossingwavelength of the Stokes- $V$ profile versus the vertical component of the magnetic field at $\tau_{500}=0.1$. a) Fe I $630.2 \mathrm{~nm}$, b) Fe I $1564.8 \mathrm{~nm}$. The lines show binned averages. On average, strong magnetic flux concentrations are associated with downflows (negative velocity), while weak fields $B<200 \mathrm{G}$ show some preference for (granular) upflows. product $\left(v_{z, \tau_{500}=0.1}-v_{z, \tau_{500}=0.01}\right) \cdot\left(\left|B_{z}\right|_{\tau_{500}=0.1}-\left|B_{z}\right|_{\tau_{500}=0.01}\right)$, which corresponds to the average gradients over the formation height of the lines, thus representing the relevant quantity in Eq. (8). It is clear that the sign of the average asymmetry is in accordance with the prediction from this relation. The scatter results from the fact that the quantity considered in Fig. 19 represents average gradients over an extended height range and thus does not take into account local variations within the height range of line formation. The numerous points with negative area asymmetry in Fig. 19 in most cases correspond to very weak Stokes- $V$ profiles formed over granules; they give only a minor contribution to the asymmetry of the average profile.

The wavelength shift of the Stokes- $V$ zero crossing has often been used to determine the velocity of magnetized plasma in observations (e.g., Solanki 1986; Grossmann-Doerth et al. 1996; Martínez Pillet et al. 1997; Sigwarth et al. 1999). The $630.2 \mathrm{~nm}$ and $1564.8 \mathrm{~nm}$ lines are formed at somewhat different heights in the solar atmosphere, so that they trace the velocity field along the line of sight in a different way. The $1564.8 \mathrm{~nm}$ line is formed deeper, where velocities typically are larger. For the simulated magneto-convection, Fig. 20 shows scatter plots of the lineof-sight velocity determined from the zero-crossing wavelength of the synthetic Stokes- $V$ profiles versus the vertical magnetic field at the level $\tau_{500}=0.1$. The binned averages given by the line show that downflows (negative velocity) dominate for field strength above a few hundred Gauss, with the stronger flows typically shown by the infrared line. For field strength around one kilogauss we find average downflows of the order of $1 \mathrm{~km} \mathrm{~s}^{-1}$ with the $630.2 \mathrm{~nm}$ line (cf. Sigwarth et al. 1999, their Fig. 6) while the deeper originating $1564.8 \mathrm{~nm}$ shows about $2 \mathrm{~km} \mathrm{~s}^{-1}$ downflow. The weak fields are predominantly associated with granular upflows. The strongest redshifts shown by the infrared line are at field strengths in the range 500-1000 G, which are typical of the expanding field in the periphery of strong flux concentrations. For field strengths corresponding to the cores of the flux concentrations, the zero-crossing shift is reduced to about $1 \mathrm{~km} \mathrm{~s}^{-1}$. This suggests that this shift could be partly due to the entrainment of material from the surrounding granular downflow, an effect probably depending also on the spatial resolution of the simulation.

\section{Conclusions}

We have analyzed the spectro-polarimetric signatures of 3D MHD models of solar magneto-convection, based on the synthesis of Zeeman-sensitive Fe I lines in the visible and in the infrared. The comparison between various properties of the synthetic Stokes profiles (such as line strength, line width, Doppler shift, Stokes- $V$ signal, area and amplitude asymmetries) with the physical properties of the model (temperature, magnetic field, and velocities) provides insight into the mechanisms underlying various characteristics of the observed spectra. In particular, the generation of strongly asymmetric Stokes- $V$ profiles in the periphery of magnetic flux concentrations largely confirms earlier simplified models based upon lines of sight crossing the magnetopause of flux concentrations fanning out with height. The line weakening in strong magnetic fields is found to be related to larger temperature (and smaller temperature gradient) in the simulated magnetic flux concentrations, in agreement with earlier interpretations of observational results.

Acknowledgements. The authors are grateful to Sanja Danilovic for test calculations of spectral line profiles.

\section{References}

Asplund, M., Nordlund, Å., Trampedach, R., Allende Prieto, C., \& Stein, R. F. 2000a, A\&A, 359, 729

Asplund, M., Nordlund, Å., Trampedach, R., \& Stein, R. F. 2000b, A\&A, 359, 743

Bard, A., Kock, A., \& Kock, M. 1991, A\&A, 248, 315

Beckers, J. M., \& Milkey, R. W. 1975, Sol. Phys., 43, 289

Bellot Rubio, L. R., \& Borrero, J. M. 2002, A\&A, 391, 331

Bellot Rubio, L. R., Ruiz Cobo, B., \& Collados, M. 1997, ApJ, 478, L45

Borrero, J. M., Bellot Rubio, L. R., Barklem, P. S., \& 1 Toro Iniesta, J. C. 2003, A\&A, 404, 749

Borrero, J. M., Solanki, S. K., Lagg, A., Socas-Navarro, H., \& Lites, B. 2006, A\&A, 450, 383

Cabrera Solana, D., Bellot Rubio, L. R., \& Del Toro Iniesta, J. C. 2005, A\&A, 439, 687

Chapman, G. A., \& Sheeley, Jr., N. R. 1968, Sol. Phys., 5, 442

Del Toro Iniesta, J. C. 2003, Introduction to Spectropolarimetry (Cambridge/UK: Cambridge University Press)

Del Toro Iniesta, J. C., Tarbell, T. D., \& Ruiz Cobo, B. 1994, ApJ, 436, 400

Delbouille, L., Roland, G., \& Neven, L. 1973, Atlas photometrique du spectre solaire de $\lambda 3000$ a $\lambda 10000$ (Universite de Liège, Institut d'Astrophysique) 
Frutiger, C. 2000, Ph.D. Thesis, No. 13896, ETH Zürich

Grossmann-Doerth, U., Keller, C. U., \& Schuessler, M. 1996, A\&A, 315, 610

Grossmann-Doerth, U., Larsson, B., \& Solanki, S. K. 1988a, A\&A, 204, 266

Grossmann-Doerth, U., Schüssler, M., \& Solanki, S. K. 1988b, A\&A, 206, L37

Grossmann-Doerth, U., Schüssler, M., \& Solanki, S. K. 1988c, A\&A, 206, L37

Grossmann-Doerth, U., Schüssler, M., \& Solanki, S. K. 1989, A\&A, 221, 338

Illing, R. M. E., Landman, D. A., \& Mickey, D. L. 1975, A\&A, 41, 183

Khomenko, E. V., Martínez González, M. J., Collados, M., et al. 2005a, A\&A, 436, L27

Khomenko, E. V., Shelyag, S., Solanki, S. K., \& Vögler, A. 2005b, A\&A, 442, 1059

Landi Degl'Innocenti, E., \& Landi Degl' Iinnocenti, M. 1977, A\&A, 56, 111

Lites, B. W., Leka, K. D., Skumanich, A., Martinez Pillet, V., \& Shimizu, T. 1996, ApJ, 460, 1019

Martínez Pillet, V., Lites, B. W., \& Skumanich, A. 1997, ApJ, 474, 810

Nesis, A., Hammer, R., Hanslmeier, A., et al. 1996, A\&A, 310, 973

Rabin, D. 1992, ApJ, 391, 832

Sanchez Almeida, J., Ruiz Cobo, B., \& Del Toro Iniesta, J. C. 1996, A\&A, 314, 295

Shchukina, N., \& Trujillo Bueno, J. 2001, ApJ, 550, 970

Shelyag, S. 2004, Ph.D. Thesis, University of Göttingen, Germany, http://www. solar-system-school.de/alumni/shelyag.pdf Sigwarth, M. 2001, ApJ, 563, 1031

Sigwarth, M., Balasubramaniam, K. S., Knölker, M., \& Schmidt, W. 1999, A\&A, 349,941
Socas-Navarro, H., \& Lites, B. W. 2004, ApJ, 616, 587

Solanki, S. K. 1986, A\&A, 168, 311

Solanki, S. K. 1987, Ph.D. Thesis, No. 8309, ETH Zürich

Solanki, S. K. 1989, A\&A, 224, 225

Solanki, S. K. 1993, Space Sci. Rev., 63, 1

Solanki, S. K., Inhester, B., \& Schüssler, M. 2006, Rep. Progr. Phys., 69, 563

Solanki, S. K., \& Pahlke, K. D. 1988, A\&A, 201, 143

Solanki, S. K., Rüedi, I., Bianda, M., \& Steffen, M. 1996, A\&A, 308, 623

Solanki, S. K., Rüedi, I. K., \& Livingston, W. 1992, A\&A, 263, 312

Solanki, S. K., \& Stenflo, J. O. 1984, A\&A, 140, 185

Stenflo, J. O. 1973, Sol. Phys., 32, 41

Stenflo, J. O., Solanki, S. K., \& Harvey, J. W. 1987a, A\&A, 171, 305

Stenflo, J. O., Solanki, S. K., \& Harvey, J. W. 1987b, A\&A, 173, 167

Stolpe, F., \& Kneer, F. 2000, A\&A, 353, 1094

Vögler, A. 2003, Ph.D. Thesis, University of Göttingen, Germany, http://webdoc. sub.gwdg.de/diss/2004/voegler

Vögler, A., Shelyag, S., Schüssler, M., et al. 2003, in Modelling of Stellar Atmospheres, IAU-Symp. No. 210, ed. N. E. Piskunov, W. W. Weiss, \& D. F. Gray (San Francisco: ASP), 157

Vögler, A., Shelyag, S., Schüssler, M., et al. 2005, A\&A, 429, 335

Wiehr, E. 1978, A\&A, 69, 279

Zayer, I., Solanki, S. K., \& Stenflo, J. O. 1989, A\&A, 211, 463 